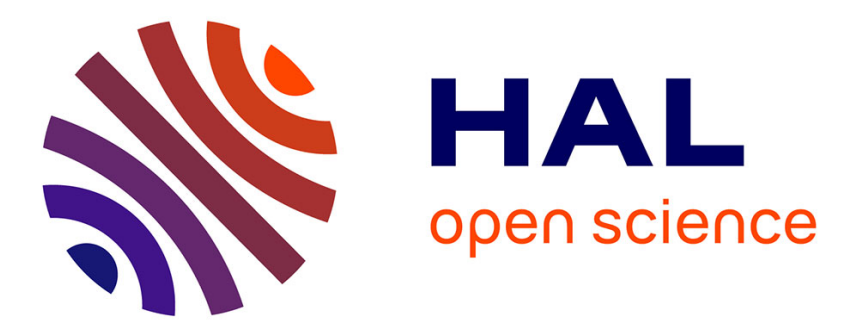

\title{
Morphological characterization and elastic response of a granular material
}

Élodie Kaeshammer, L. Borne, François Willot, Petr Dokládal, S. Belon

\section{To cite this version:}

Élodie Kaeshammer, L. Borne, François Willot, Petr Dokládal, S. Belon. Morphological characterization and elastic response of a granular material. Computational Materials Science, 2021, 190, pp.110247. 10.1016/j.commatsci.2020.110247 . hal-03115043

\section{HAL Id: hal-03115043 \\ https: / hal-mines-paristech.archives-ouvertes.fr/hal-03115043}

Submitted on 20 Jan 2021

HAL is a multi-disciplinary open access archive for the deposit and dissemination of scientific research documents, whether they are published or not. The documents may come from teaching and research institutions in France or abroad, or from public or private research centers.
L'archive ouverte pluridisciplinaire HAL, est destinée au dépôt et à la diffusion de documents scientifiques de niveau recherche, publiés ou non, émanant des établissements d'enseignement et de recherche français ou étrangers, des laboratoires publics ou privés.

$$
\text { Copyright }
$$




\title{
Morphological characterization and elastic response of a granular material
}

\author{
É. Kaeshammer ${ }^{\mathrm{a}, \mathrm{b}, \mathrm{c}}$, L. Borne ${ }^{\mathrm{b}}$, F. Willote ${ }^{\mathrm{c}, \mathrm{d}}$, P. Dokládal ${ }^{\mathrm{c}}, \mathrm{S}$. Belon ${ }^{\mathrm{a}}$ \\ ${ }^{a}$ CEA DAM, 46500 Gramat, France \\ ${ }^{b}$ French-German Research Institute of Saint-Louis (ISL), 5 rue du Général Cassagnou, 68301 Saint-Louis, France \\ ${ }^{c}$ Mines ParisTech, PSL Research University, Center for Mathematical Morphology, 35 rue St Honoré, \\ 77300 Fontainebleau, France \\ ${ }^{d}$ Center for Materials, 63-65 Rue Henri Auguste Desbruères, 91100 Corbeil-Essonnes, France
}

Published in: Computational Material Science 190 (2021), 110247. DOI: 10.1016/j.commatsci.2020.110247.

\begin{abstract}
The granular structures of energetic materials made of hexogene particles embedded in a matrix are characterized using a combination of flotation, light-scattering measurements and micro-computed tomography images. The complementary nature of the three characterization techniques, when employed with this type of material, allows one to derive accurate estimates for the grain size distribution and the particle bulk density distribution. Three types of granular formulations, with the same weight fraction of particles but markedly different grains morphology and shock-sensitivity properties, are addressed. The particles granulometry and intra-granular porosities differ from one material to another. The grains spatial dispersion in the three formulations, characterized by the scale-dependent local density, also varies from one formulation to another. Specifically, we show that samples containing elongated grains display "abnormal" variance scalings for the grains local density, as a result of long-range correlations in the granular packing structure. The use of virtual microtructures to predict the materials mechanical properties is addressed and discussed.
\end{abstract}

Keywords: Flotation, Light-scattering measurement, Fourier methods, Granular structures, Virtual microstructures.

\section{Introduction}

Granular media, made of assemblies of densely-packed grains, may be regarded as discrete systems of particles that interact with one another. They display a large variety of mechanical behaviors (Guyon et al., 1990), owing to highly-nonlinear contact mechanisms between particles (Kaneko et al., 2003), avalanche phenomena (Peña et al., 2009) and instabilities. Continuum mechanics and homogenization theories (Milton, 2002) may be used to tackle more simple problems and predict quasi-static transport properties (Xu et al., 2018). Even then, accurate mechanical predictions require, in general, an intimate knowledge of the grains packing structure, and of the geometrical factors that monitor the grains shape and spatial dispersion, or their connectivity (Jeulin, 2002). As a straightforward example, in the case where grains are much stiffer than the embedding matrix, the effective properties of the granular structure depends on the percolation of the grain packing (Torquato, 2013). Such geometrical effects are difficult to quantify in real materials, which are random, and must be described by probabilistic models (Stoyan et al., 1995). 
Numerical approaches combining full-fields mechanical computations and "virtual materials" (Gaiselmann et al., 2014; Wang et al., 2015) based on random probabilistic models (Stoyan et al., 1995) or machine learning methods, are an efficient method for assessing microstructure-property relationships (Stenzel et al., 2016; Wu et al., 2018). Even then, thorough characterization techniques combining imaging, mathematical morphology and stereology (Russ and Dehoff, 2012) are required to construct random models. This approach is not straightforward, however, as predictions generally depend on the choice of morphological criteria used to fit the parameters of virtual materials (Neumann et al., 2020) and validation of the predictions require a large number of experimental data (Neumann et al., 2020). In the present work, our validation of a stochastic virtual material is based not only on the predicted effective mechanical properties, but also on the material's local response.

The present article is devoted to the study of three explosive materials provided by the FrenchGerman research Institute of Saint-Louis (ISL). An explosive material is a material able to release a great quantity of energy in a small amount of time when thermal thresholds are exceeded. Each of the three materials is based on RDX organic compound also known as Hexogen $\left(\mathrm{C}_{3} \mathrm{H}_{6} \mathrm{~N}_{6} \mathrm{O}_{6}\right)$, assembled as a granular set of particles embedded in a wax matrix. The first sample contains commercial Reduced Sensitivity Hexogen grains (RS-RDX) which present numerous defects such as solvent inclusions, pores, micro-cracks and twinned grains. The second material is made of particles noted Raw Very Insensitive Hexogen (RVI-RDX) obtained after a recrystallization of the RS-RDX particles (Borne, 2006). These particles present sharp edges while crystallization significantly reduces the number of intra-granular defects (Fig. 1b, top-left). The third material is made of the ISL patented Very Insensitive Hexogen (VI-RDX) particles with smooth shape and few internal defects, obtained after a surface treatment of RVI-RDX particles. Intra-granular defects are well-known to increase the sensitivity to shock of energetic materials. The operations of recrystallization and surface treatment aim to reduce the sensitivity to shock of RDX based material, and so, to increase the security by limiting accidental initiation of the explosive. All manufactured samples are made of $70 \%$ mass fraction of RDX particles, and therefore have the same composition and the same formulation density.

The materials studied in the present work have very different shock sensitivity properties. Experimental studies have shown that shock sensitivity is driven by microstructural parameters, notably the size of explosive particles (Moulard et al., 1985), their shape (van der Steen et al., 1989), extra-granular (Mishra and Vande Kieft, 1988) and intra-granular defects (Borne, 1993; Baillou et al., 1993; Borne, 1998; Borne and Beaucamp, 2002). In the present work, we seek to determine if the quasi-static properties of the same material also exhibit differences, and if these differences can be correlated to the microstructure.

The present work is organized as follows. Sec. (2) is devoted to the morphological characterization obtained from the micro-computed tomography $(\mu \mathrm{CT})$ images. It also gives a brief description of the filtering and segmentation techniques applied to the $\mu \mathrm{CT}$ images. Emphasis is put on the variance properties of the local grains density as a way to evaluate the representativity of the images. Grain size-distribution and spatial covariance are considered. These measurements are then compared to experimental data obtained by flotation and light-scattering measurements. The effective and local elastic response is studied in Sec. (3) making use of full-field Fourier-based numerical computations. Virtual microstructures are introduced in Sec. (4). Their adequacy for 
predicting the mechanical properties is discussed. We conclude in Sec. (5).

\section{Morphological characterization based on micro-computed tomography images}

\subsection{Image processing and $3 D$ segmentation}

Three cylindrical samples with a height of $10 \mathrm{~mm}$ and a radius of $5 \mathrm{~mm}$ are imaged at the CEA Gramat by micro-computed tomography $(\mu \mathrm{CT})$ on a Skycan 1172 device, revealing their inner microstructures, in three dimensions (Figs. 1a-c). The resolution is set to $3.67 \mu \mathrm{m}$ for materials based on RS-RDX and RVI-RDX grains (Figs. 1a-b) and to $3.54 \mu \mathrm{m}$ for VI-RDX samples (Fig. 1c). To separate neighbouring particles from each other in X-ray micro-tomography images, we use a combination of standard filtering and watershed transforms (Beucher, 1994) and morphological criteria relevant to granular structures, which are briefly described below. The reader is referred to (Chabardès et al., 2017) for a detailed discussion of these segmentation techniques applied to grains reconstruction.

An adaptive recursive bilateral filter (Yang, 2012) is first applied as a way to remove noise in the images. The bilateral filter is an edge-preserving filter that acts in a similar way to the Gaussian filter but introduces less blur. It accepts two kernels: a spectral (or range) kernel and a spatial kernel that together control the intensity of denoising. Intituevely, a voxel from one phase (e.g. the RDX grains) should be less affected by a voxel from the other phase (the binder). The range kernel adapts to the noise and to the difference of the intensity of the different phases. Similarly, the spectral kernel is used to filter inside the phases without blurring across the particle borders. As a result, the spectral kernel size should be no wider than the intensity difference of the phases, and the spatial kernel size should be no wider than the expected particle size. The reader is referred to (Chabards, 2018) for details how to select automatically the best parameter values and an extension to multi-phase materials.

Second, a watershed transform (Beucher and Meyer, 1993) is applied to the filtered image, resulting in numerous over-segmented RDX particles. The markers used for the watershed transform are the local maxima of the distance function from the binder, which is strictly positive inside the grains. We follow (Chabardès et al., 2017) and introduce a geometric criterion based on the distance function as a way to detect bottlenecks in-between two adjacent labels, and merge particles.

The resulting segmentation algorithm, carried out with the Mines ParisTech software library SAMG (Chabards, 2018), is applied to the three sample images. Despite this treatment however, about 150 hexogen particles, comprising the most elongated and twinned grains in the RS-RDX sample, remain over-segmented. This bias is corrected by identifying and merging manually the labels in this small subset of grains. Some of the resulting segmentations are represented in Figs. (1d-f). For each of the three materials, four independent $\mu \mathrm{CT}$ volumes are used in the present study. Each image contains $1000^{3}$ voxels and represents a volume size of $3.54^{3} \mathrm{~mm}^{3}$ (RSRDX, RVI-RDX) and $3.67^{3} \mathrm{~mm}^{3}$ (VI-RDX). To simulate microstructures representative of the granular materials later on, grains are extracted from the segmented images and saved into a library of about 15,000 grain shapes (see Table 1). 


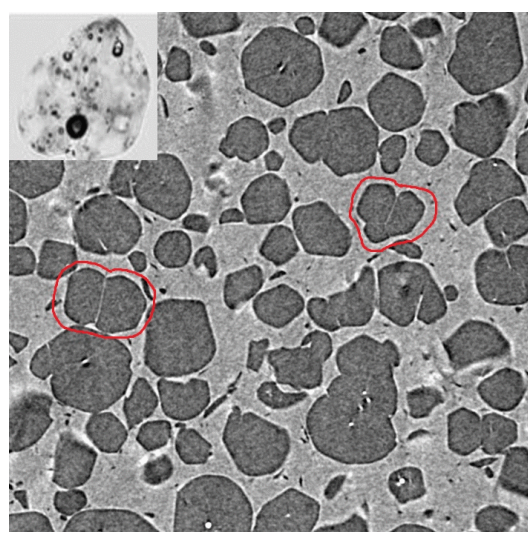

(a)

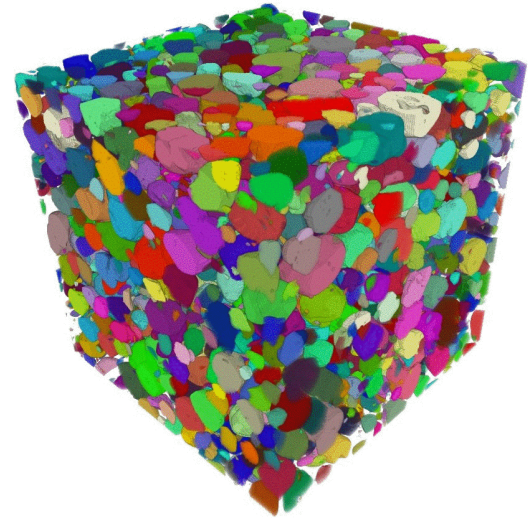

(d)

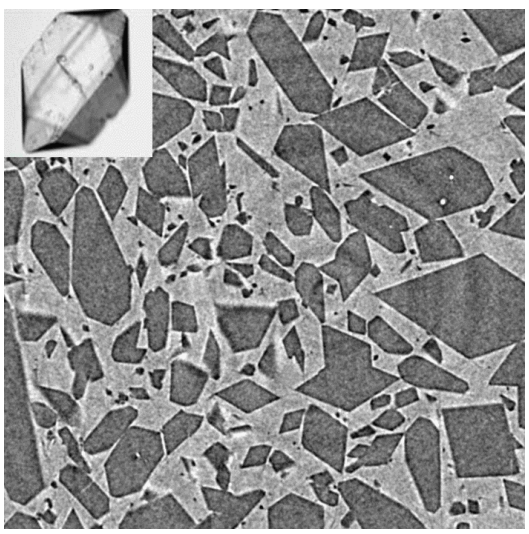

(b)

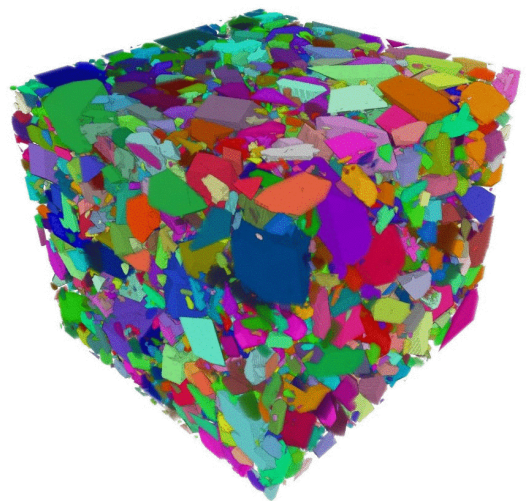

(e)

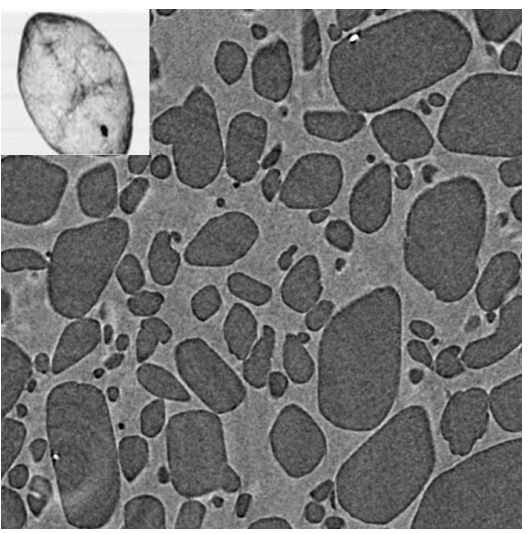

(c)

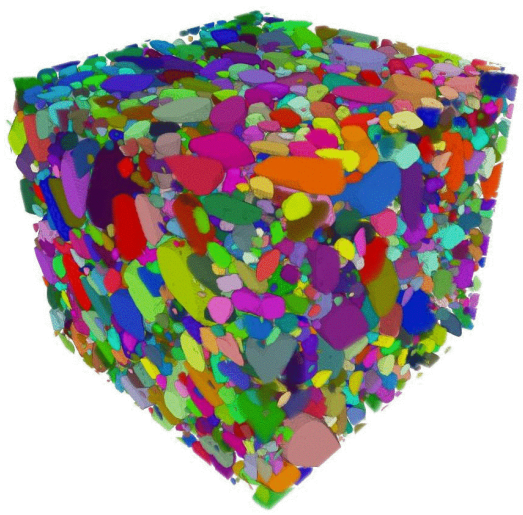

(f)

Figure 1: Micro-computed tomography images (2D sections) of energetic materials containing different types of RDX particles. (a) RS-RDX particles with twinned grains indicated in red, (b) RVI-RDX particles with sharp edges, (c) VI-RDX particle with smooth, rounded shape. (d-f) Segmented volumes. Optical images of the grains are shown as embedded images, top-left of (a-c).

\subsection{Representativity of the segmented images}

According to experimental data, the RDX weight fraction is $70 \%$ in all materials, which amounts to a volume fraction of $p \approx 56 \%$. This is in excellent agreement with the volume fraction of RDX grains as measured in images (Table 1) and suggest even the small grains are present in the segmented volumes. Nevertheless, the segmented images represent only a small subvolume of the cylindrical specimens. For the former to be considered as "representative" of the later, two conditions must hold. First, the granular microstructures must be stationary (i.e. translationinvariant). This property is assumed here. Second, the size of the imaged volume should be large compared to the typical size of the heterogeneities, grains, or arrangement of grains, present in the grain-packing structure. To determine if the segmented images are representative of the granular materials, we make use of the Matheron's theory (Matheron, 1989; Lantuéjoul, 1991), which we briefly describe hereafter.

Let us denote $\langle Z(x)\rangle_{V}$ the mean over a volume $V$ of a spatial quantity $Z(x)$ and $\langle Z(x)\rangle$ the 
Table 1: Number of grains extracted from the segmented images and mean volume fraction, for the three types of granular materials.

\begin{tabular}{l|ccc} 
& RS-RDX/Wax & RVI-RDX/Wax & VI-RDX/Wax \\
\hline Number of grains & 13623 & 22147 & 25212 \\
\hline Volume fraction & $55.6 \%$ & $56.2 \%$ & $55.7 \%$ \\
\hline
\end{tabular}

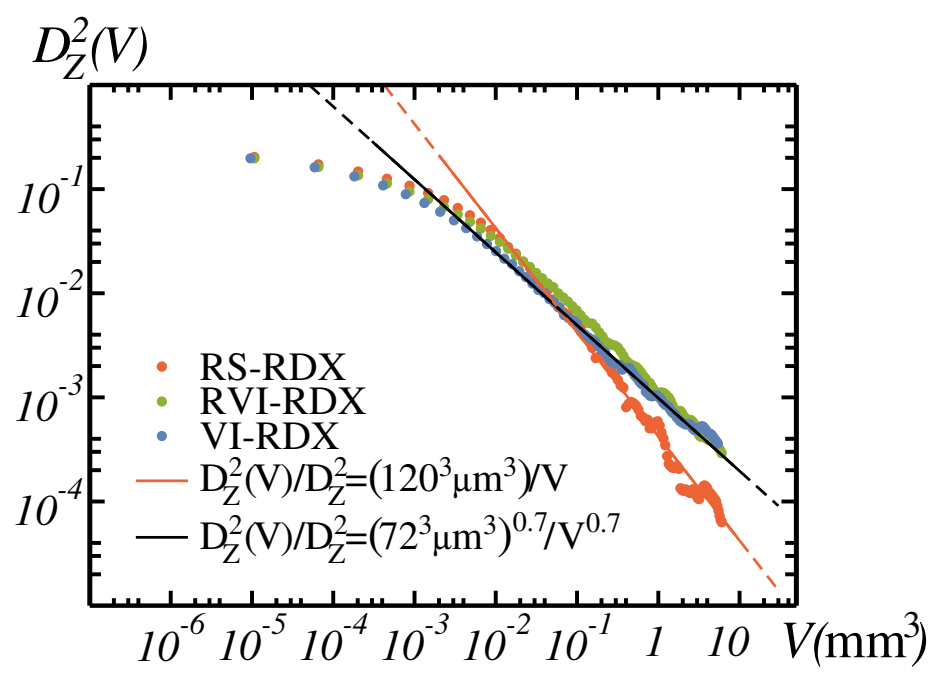

Figure 2: Average variance $D_{Z}^{2}(V)$ vs. subvolume size $V$ for the three materials and corresponding powerlaw fit with $\alpha=1$ or $\alpha=0.7$.

mean of $Z$ over $\mathbb{R}^{3}$. The quantity $\langle Z(x)\rangle_{V}$ is, for fixed volume size $V$, a random variable with mean $\langle Z(x)\rangle$ and variance $D_{Z}^{2}(V)$. For a stationary, ergodic random set, $D_{Z}^{2}(V) \rightarrow 0$ as $V \rightarrow \infty$. Matheron provides the more precise asymptotic expansion (Matheron, 1989):

$$
D_{Z}^{2}(V)=D_{Z}^{2} \frac{A_{3}}{V}+o(1 / V), \quad V \gg A_{3},
$$

which relates the variance $D_{Z}^{2}(V)$ with the point-variance $D_{Z}^{2}=\left\langle Z(x)^{2}\right\rangle-\langle Z(x)\rangle^{2}$ and the integral range (homogeneous to a volume):

$$
A_{3}=\frac{1}{D_{Z}^{2}} \int_{h \in \mathbb{R}^{3}}\left(\langle Z(x) Z(x+h)\rangle-\langle Z(x)\rangle^{2}\right) d h .
$$

These expressions generalize to any self-averaging quantity $Z(x)$, however, for simplicity we assume hereafter that $Z(x)$ is the grains characteristic function so that $\langle Z(x)\rangle_{V}$ is the grains volume fraction, estimated over a volume $V$. The integral range $A_{3}$ now reads:

$$
A_{3}=\frac{1}{D_{Z}^{2}} \int_{h \in \mathbb{R}^{3}}[C(h)-p] d h, \quad D_{Z}^{2}=p(1-p),
$$

where $p=\langle Z(x)\rangle$ is the volume fraction of the grains and $C(h)=P\{Z(x)=1, Z(x+h)=1\}$ is the covariance function, defined by the probability that two points separated by a vector $h$ are 
Table 2: Values of the exponent, reference volume and point variance in Eq. (4) for the three types of granular materials, and relative error on the grain volume fraction as measured in images.

\begin{tabular}{l|ccc} 
& RS-RDX/Wax & RVI-RDX/Wax & VI-RDX/Wax \\
\hline$\alpha$ & 1.0 & 0.75 & 0.7 \\
$V_{0}\left(\mu^{3}\right)$ & $120^{3}$ & $93^{3}$ & $72^{3}$ \\
$D_{Z}^{2}$ & 0.247 & 0.246 & 0.247 \\
$\varepsilon_{\text {rel }}(\%)$ & 0.58 & 1.41 & 1.50
\end{tabular}

in the grains. The integral range may be infinite $\left(A_{3}=+\infty\right)$ in materials exhibiting correlations at infinite length scales, in which case Eq. (1) does not apply, and must be replaced by a new scaling law. In the limiting case of a Boolean set of cylinders with infinitely-large aspect ratios, the variance of the volume fraction scales as (Jeulin, 2016):

$$
D_{Z}^{2}(V)=D_{Z}^{2}\left(\frac{V_{0}}{V}\right)^{\alpha}, \quad V \rightarrow \infty,
$$

where $V_{0}$ is a reference volume. The exponent $\alpha<1$ indicates a slower decay of the variance in this case as compared to (4) and depends on the object type $(\alpha=1 / 3$ and $2 / 3$ for oblate and prolate cylinders). The factor $V_{0}$ plays a similar role as the integral range. In a Boolean set of cylinders with large aspect ratio $b / a \gg 1$, where $b$ is the largest dimension and $a$ the smallest, expansion (4) occurs in the range $V \ll b^{3}$ while (1) is recovered in the domain $V \gg b^{3}$ (Willot, 2017).

The variance $D_{Z}^{2}(V)$ is evaluated by dividing the segmented $\mu \mathrm{CT}$ images into subvolumes of size $V$ and computing the variance of the apparent grain density. For a given granular material (RS-RDX, RVI-RDX, VI-RDX), the data is averaged over all sample images (Fig. 2, in log-log plot). A powerlaw fit of the data is determined in a region of interest of nearly 3 decades. The scaling law obtained is consistent with Eq. (4), where $D_{Z}^{2} \approx 0.25$ for all materials, whereas the exponent $\alpha$ and reference volume $V_{0}$ take on different values for each granular packing (2). The exponents $\alpha \approx 0.7$ and $\alpha \approx 0.75$ are indicative of strong finite-size effects and of the presence of long-range correlations in the RVI-RDX and VI-RDX grain packing types. At this point, it is useful to note that RVI-RDX and VI-RDX grains have higher aspect ratio than RS-RDX grains, as will be quantified later on. A wide granulometry distribution with slowly-decaying tail may result in an asymptotic expansion of type (4), with $\alpha<1$ (see appendix Appendix A, where this result is derived in the case of an ideal Boolean model). The granulometry distributions for the RS-RDX, RVI-RDX and VI-RDX based materials will be accordingly compared in the next section.

The relative error on the grain volume fraction, also indicated in Table (2), is given by:

$$
\varepsilon_{r e l}=\frac{2 D_{Z}(V)}{p \sqrt{n}}=2 \sqrt{\frac{(1-p) V_{0}^{\alpha}}{p n V^{\alpha}}},
$$

computed $n=4$ volumes, each of size $V=1000^{3}$ voxels. The above equation may also be used to determine the volume required to obtain a given relative error. For instance, a volume of size of $3.8^{3}, 9.0^{3}$ and $10^{3} \mathrm{~mm}^{3}$ (i.e. $1030^{3}, 2,530^{3}$ and $2,820^{3}$ voxels) is necessary to measure the grains volume fraction with a relative error of $1 \%$, for the RS-RDX, RVI-RDX and VI-RDX material respectively. 


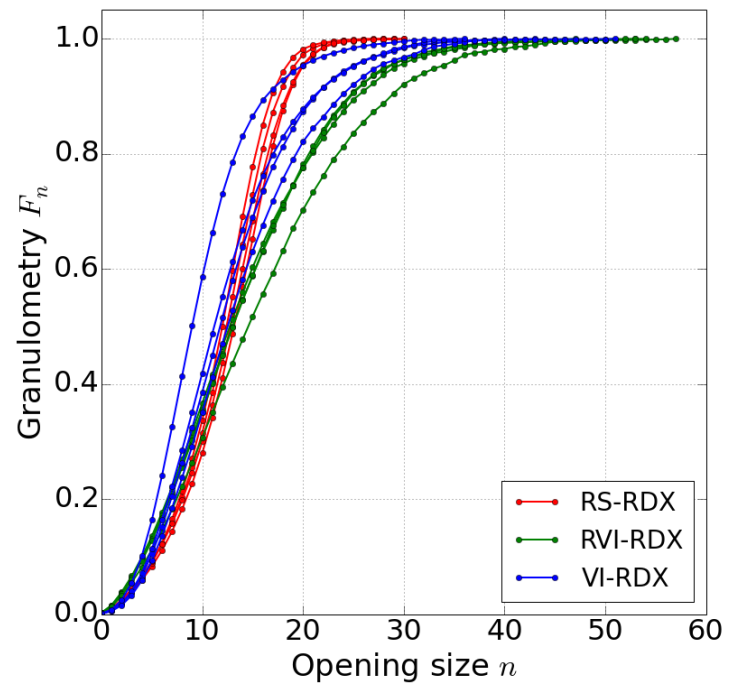

(a)

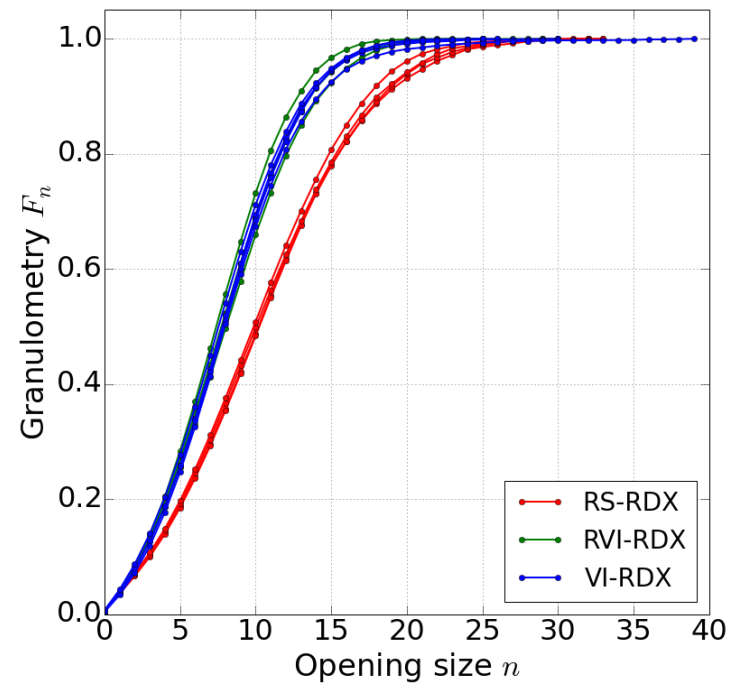

(b)

Figure 3: Morphological granulometry by openings of (a) the RDX grains and (b) the wax binder, for the four segmented volumes of each material.

\subsection{Morphological granulometry and spatial covariance}

The morphological granulometry by opening $\gamma$, i.e. a sequence of dilation of erosion with a structuring element of an increasing dimension, is considered to extract the characteristic sizes of a subset $X \subset \mathbb{R}^{3}$, which may be the RDX grains or its complementary set (Serra, 1982). With an increasing structuring element the opening progressively removes larger grains. The granulometric curve, equivalent to a cumulative probability distribution $F_{n}$, is obtained as:

$$
F_{n}(X)=1-\frac{\left|\gamma_{n}(X)\right|}{\left|\gamma_{0}(X)\right|}
$$

where $\left|\gamma_{n}(X)\right|$ denotes the volume of the opening $\gamma_{n}$ of set $X$, the subscript $n$ refers to the size of the structuring element, and $\gamma_{0}(X)=X$. A cubic structuring element is used.

The granulometry for the RDX grains and for the binder for the four segmented volumes of each material are represented in Figs. (3). Small variability is observed within different samples of the same type of material. Moreover, different cumulative distributions are obtained for the three types of materials. The RDX distribution curves for the RVI-RDX and VI-RDX samples are shifted to the right compared to RS-RDX, showing that recrystallization increases the grains' size. The granulometric curve obtained for the RS-RDX sample is much narrower than that observed for the VI-RDX and RVI-RDX samples. This is consistent with the slow decay of the variances for these two materials as compared to the former (see Table 2).

The granulometric curves relative to the binder, represented in Fig. (3b), show small variability within samples of the same material. The granulometric curves are similar for the RVI-RDX and 


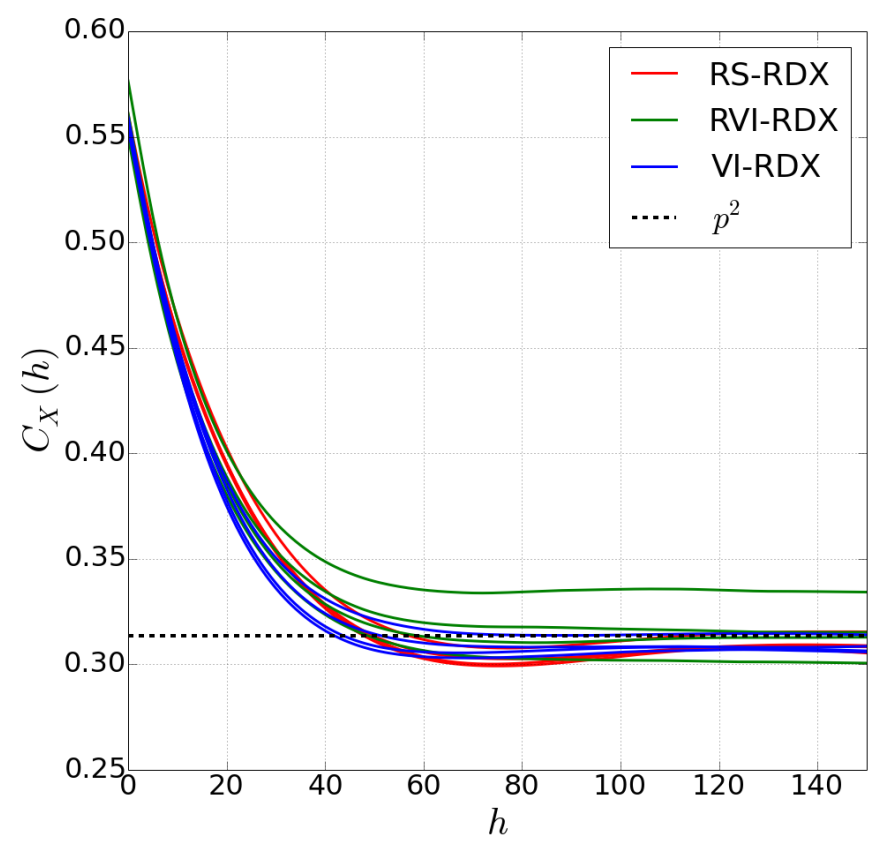

Figure 4: Spatial covariance of the RDX grains for the four segmented volumes for each material. The plateau $p^{2}$ is plotted in dashed black line with $p$ equal to experimental RDX volume fraction of 0.56.

VI-RDX materials. That of the RS-RDX material is wider in comparison. The presence of large empty regions in this case suggests that RDX grains are less homogeneously distributed in space in the RS-RDX material, although other factors, such as the non-convexity of many of the RS-RDX grains, must also play a role.

The spatial covariance $C_{X}(h)$, defined as the probability that two points separated by a distance $h$ belongs to set $X$, is represented in Fig. (4), for each of the segmented volumes. The volume fraction of the grain is recovered at the point $h=0(C(0)=p)$, whereas the slope at the origin is proportional to the specific surface area. When $h$ is large, the covariance approaches the limiting value $C(h) \approx p^{2}$. The spatial covariances of different volumes for the same material are similar. For instance, the Pearson's correlation coefficient calculated between the four volumes for the RS-RDX and VI-RDX are 0.9999 and 0.9987 respectively. The spatial covariance between all three materials are quite similar as well, with a correlation coefficient of at least 0.9928 between the RS-RDX and VI-RDX materials, suggesting that the covariance is unable to distinguish between the different grain shapes. Interestingly, the covariance function reaches the value $p^{2}$ at roughly $h=50$ pixels, which is somewhat larger than the largest sizes in the granulometry curves (Figs. 3), highlighting the presence of correlations between distant points. Such correlations typically appear as a result of repulsion effects in models of hard-particles that forbid particles interpenetration (Torquato, 2013, Sec. 2.2). 


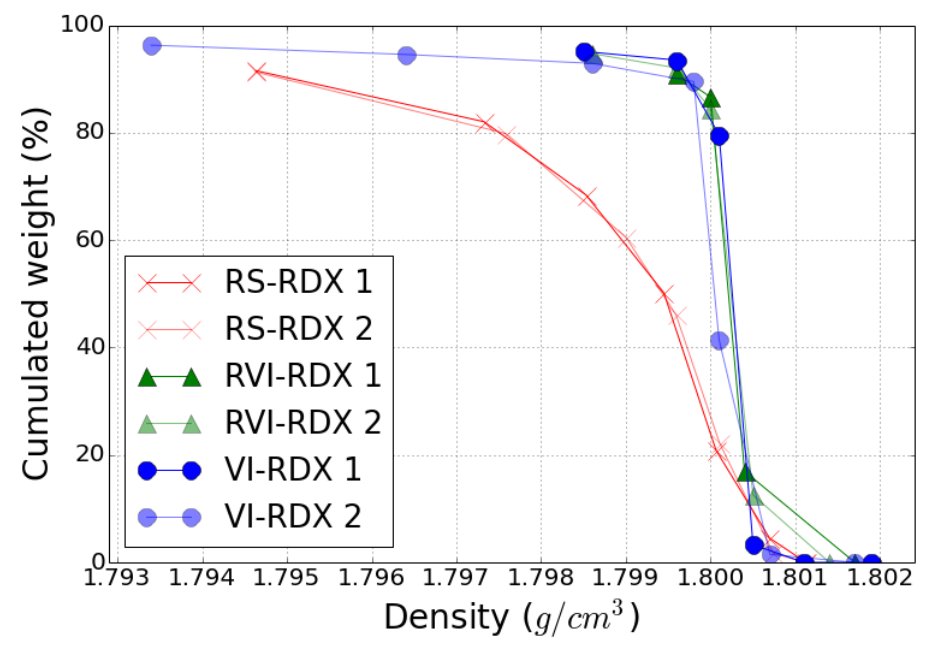

Figure 5: Experimental density of RS-RDX, RVI-RDX and VI-RDX particles.

\section{Comparison between morphological measurements and experimental data}

In this section, we compare the segmentation obtained in Sec. (2.1) with the experimental data (provided by ISL) relative to the granulometry and bulk density distributions of RDX grains which characterize the amount of pores trapped in the particles.

\subsection{Flotation and bulk density distribution}

The first characteristic is the bulk density. The bulk density gives information on defects contained within RDX grains. Bulk densities smaller than $\rho_{\max }=1.801 \mathrm{~g} / \mathrm{cm}^{3}$ reveal the presence of closed porosities or solvent inclusions. The particle bulk density is measured by the ISL patented flotation method (Borne and Patedoye, 2009). The RDX particles are immersed in a liquid mixture whose density is tuned and measured very accurately. After a dispersion of the particles in the fluid by stirring, they are separated by decantation. The heaviest particles, at the bottom, are extracted, and weighted. These steps are repeated until all the particles of RDX are extracted (Borne et al., 1999; Borne and Beaucamp, 2005). To ensure the reliability of the results, the experiment is performed twice on the same batch. Experiments are performed on $7 \mathrm{~g}$ mass particle sample, equivalent to about one million particles.

The effect of the recrystallization is clearly visible when the cumulative bulk density distribution of the RS-RDX particles is compared with that of the recrystallized particles (Fig. 5). As RS-RDX particles contain a lot of intragranular defects, their bulk density is often below the theoretic density of RDX particles $\left(1.801 \mathrm{~g} / \mathrm{cm}^{3}\right)$. On the contrary, the recrystallized particles of RVI-RDX and VI-RDX contain almost no intragranular defects so that most of the grains have a bulk density near the theoretic bulk density of RDX grains. We also notice that the cumulative bulk density distributions of the RVI-RDX and VI-RDX particles are very similar. This is due to the fact that VI-RDX particles are obtained by applying a surface treatment on RVI-RDX grains which has no effect on the intragranular defects and so on the bulk density of the particles. 


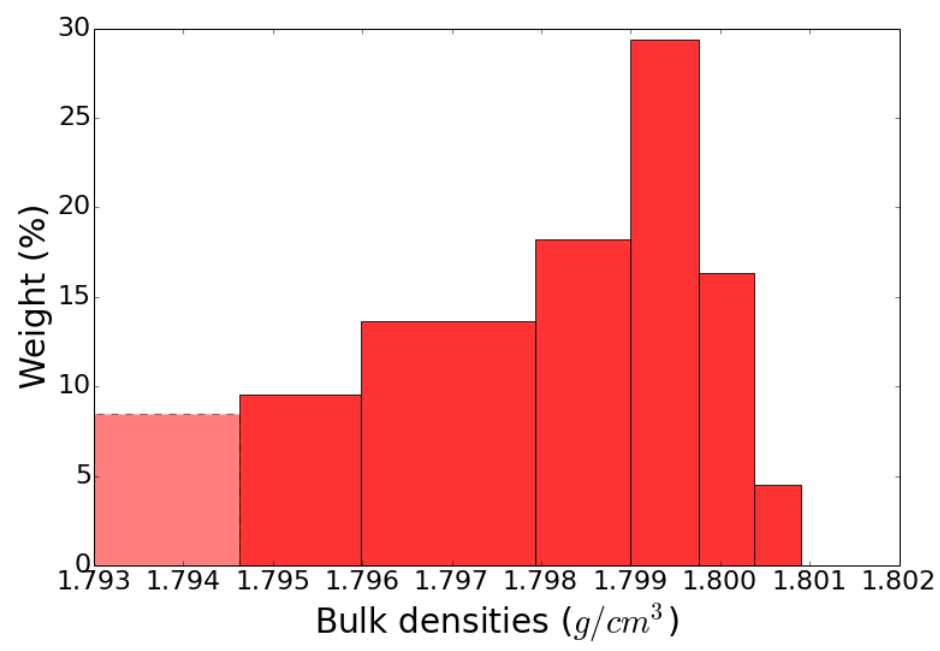

Figure 6: Classes defined with the experimental data for the first experiment on the RS-RDX grains.

These results are now used to determine the total volume of porosities for each sample. In the following we assume two opposite hypothesis: either all porosities are voids (zero density), or they are pure solvent inclusions (density of $1.0 \mathrm{~g} / \mathrm{cm}^{3}$ ).

Making use of experimental results of flotation we now define classes. For instance, the first experiment on RS-RDX particles is made of 7 points of acquisition which define 6 classes (Fig.6). The first class is composed of particles with a bulk density inbetween $1.8007 \mathrm{~g} / \mathrm{cm}^{3}$ and $1.8011 \mathrm{~g} / \mathrm{cm}^{3}$ and represents $4.54 \%$ of the total weight of the tested sample (Fig 6). Under the hypothesis that the particles are uniformly dispersed in each class $j$, one may calculate the mean bulk density $\rho_{j}$ of each class as:

$$
\frac{2}{\rho_{j}}=\frac{1}{\rho_{i-1}}+\frac{1}{\rho_{i}},
$$

where $\rho_{i}$ is the bulk density of the experimental point of acquisition $i$. The bulk density of some of the particles, ranging between $3.7 \%$ (relative to the second experiment on VI-RDX) and $9.1 \%$ in weight (relative to the first experiment on RVI-RDX), could not be measured. The missing grains are those grains with a high porosity and a bulk density lower than that of the fluid at the end of the experiment. These particles constitute a new class, defined in two different ways. The first method assigns the experimentally measured minimal bulk density to all remaining grains. For instance, in the case of the first experiment relative to RS-RDX, the class with the missing particles is made of those particles with a bulk density $\rho_{j}<1.7946 \mathrm{~g} / \mathrm{cm}^{3}$ and represent $8.44 \%$ of the total weight (Fig 6). The volume of intra-granular defects in this class is thus underestimated. In the second method, use is made of the $\mu \mathrm{CT}$ images. The segmented grain with the lowest bulk density is identified and used to define the lowest bound of the new class. The upper bound is given by the last acquisition point from the flotation experiment. The porosity is determined for each class, according to the two methods, using:

$$
\Phi_{j}=\frac{\rho_{\max }-\rho_{j}}{\rho_{\max }-\rho_{\text {incl }}}
$$


Table 3: Total volume fraction of intra-granular defect (in \%) determined with method 1 (flotation), method 2 (flotation and image analysis). The determination with method 1 only use the experimental data from the flotation. The determination with method 2 is a combination of the flotation experiment results and the $\mu \mathrm{CT}$ images analysis results in order to obtained a better accuracy.

\begin{tabular}{l|cccccc} 
& \multicolumn{2}{|c}{ RS-RDX } & \multicolumn{2}{c}{ RVI-RDX } & \multicolumn{2}{c}{ VI-RDX } \\
Defects filled with... & air & solvent & air & solvent & air & solvent \\
\hline Method 1 exp. 1 & 0.126 & 0.284 & 0.0409 & 0.0919 & 0.0474 & 0.107 \\
Method 1 exp. 2 & 0.126 & 0.284 & 0.0452 & 0.102 & 0.0694 & 0.156 \\
Mean for method 1 & 0.126 & 0.284 & 0.0430 & 0.0968 & 0.0584 & 0.131 \\
\hline Method 2 exp. 1 & 0.460 & 0.591 & 0.183 & 0.229 & 0.135 & 0.189 \\
Method 2 exp. 2 & 0.471 & 0.601 & 0.126 & 0.177 & 0.131 & 0.207 \\
Mean for method 2 & 0.465 & 0.596 & 0.155 & 0.203 & 0.133 & 0.198 \\
\hline \hline Images analysis & \multicolumn{2}{|c}{0.403} & \multicolumn{2}{c}{0.090} & 0.119
\end{tabular}

Finally, the total volume of intra-granular defects is determined. These results are compared with the total volume fraction obtained with image analysis on the segmented $\mu \mathrm{CT}$ volumes (Tab. 3).

The results, summarized in Tab. (3), indicate that the two experiences yield different predictions with respect to the inner cavity within the VI-RDX grains. More experimental points in the second experience are available than in the first experience relative to low-density particles. Under the hypothesis that all defects are porosities, the volume increases from $0.047 \%$ to $0.069 \%$ (column 5, rows 1 and 2, Table 3). These results highlight the importance of the last experimental point as the total volume of cavities depends on the precision achieved at low density. The volume of defects determined with both experimental and image analysis results (method 2) is around twice more important compared to the highest estimate obtained with method 1 . In fact, the use of image analysis in method 2 greatly reduces the discrepancy between the two experimental results (see columns 5-6, rows 4 and 5, to be compared with rows 1 and 2). Overall, the results suggest that method 2 is the most accurate. Note that image analysis results for the inner cavity, obtained from the micro-tomography images solely (row 7, Table 3) do not fall within the range measured experimentally, as defects smaller than about $1 \mu \mathrm{m}$ cannot be resolved in the images, underestimating the total volume of cavities. Flotation experiments can detect these submicronic intra-granular defects. In effect, the combined use of flotation experiment and image analysis allows one to determine the intra-granular defects with highest accuracy, whereas any of these methods, when use together, give underestimated predictions. As expected, the volume of defects for VIRDX (or RVI-RDX) is $50 \%$ less that of RS-RDX, highlighting the important role of recrystallization on this property.

The bulk density cumulative distributions are plotted in Figs. (7) for the different materials. Dots represent experimental results whereas image analysis predictions are shown as colored solid lines.

Previous experimental studies have shown that closed cavities present during the crystallization process can contain air, solvent, or both Borne et al. (1999). Thus, image analysis results are derived under two assumptions: voids are filled either with air or with a solvent of $1 \mathrm{~g} / \mathrm{cm}^{3}$. Image analysis data are in better agreement with experimental data under the hypothesis that voids are 


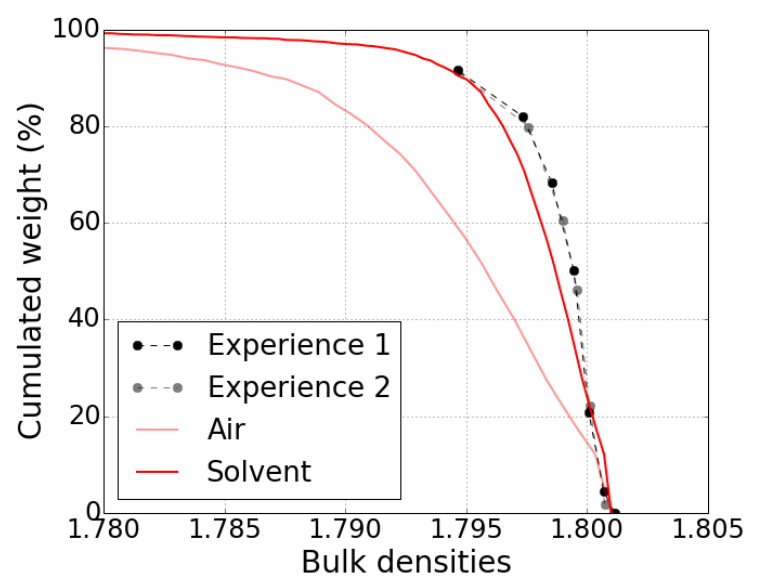

(a)

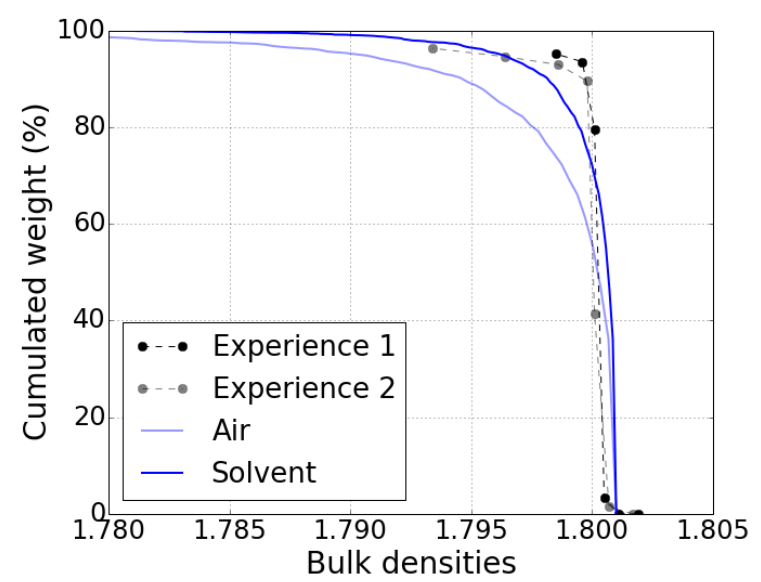

(b)

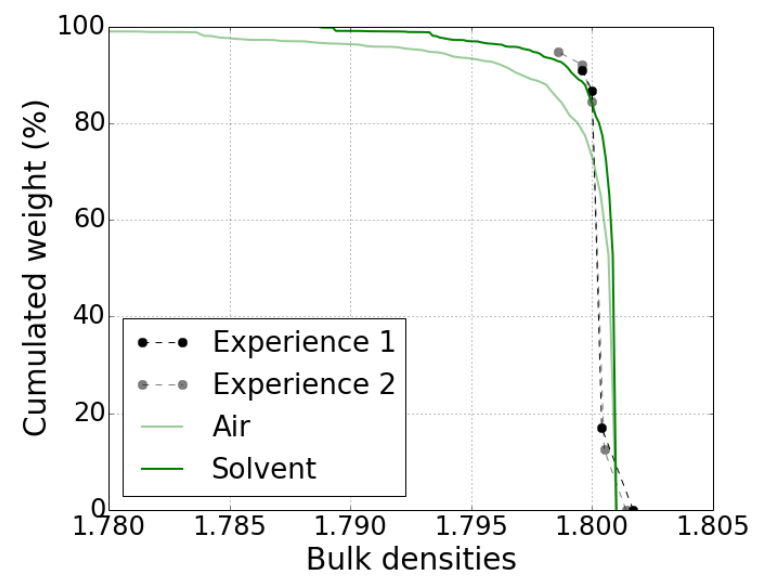

(c)

Figure 7: Comparison of experimental density and image analysis density with the hypothesis that the voids are filled with air or solvent of $1 \mathrm{~g} / \mathrm{cm}^{3}$ for (a) RS-RDX, (b) RVI-RDX and (c) VI-RDX.

filled with solvent, not air. Comparing the 3 materials, RS-RDX particles clearly contain much more pores than the two other materials. We recall that the resolution of the images (3.54 and $3.67 \mu \mathrm{m})$ prevent us from taking into account voids smaller than $3 \mu \mathrm{m}$ in the image analysis results, unlike results obtained by the flotation method.

\subsection{Light-scattering measurements and granulometry}

We now examine the granulometry distribution of particles and compare experimental results with that obtained using image analysis. The size distributions of particle diameters have been measured with Static Light Scattering (SLS) technique by a COULTER LS230 at ISL. This technique provides us with diffraction figures. Thus, the SLS-measured diameter is that of a sphere with closest diffraction figure, whereas image analysis provide a mean and equivalent diameters. The mean diameter is twice the mean distance between the center of mass and the surface of the 
particle. The equivalent diameters in volume (or surface) are the diameters of equivalent spheres (or disks) with respect to volume (or surface). The experimental measurements are performed on a sample made of $450 \mathrm{mg}$ of RDX whereas grains extracted from the four $1000^{3}$ voxels segmented volumes, totalling about $125 \mathrm{mg}$, is assumed to be representative.

The distributions of diameters provided by the COULTER LS230 device are given in Fig. (8). Care must be taken with the shapes of the distribution curves. These are smoothed result curves provided by the SLS device and the associated software. A new curve, without the smoothing effect, is recalculated with SLS raw data and used to compare experimental and image analysis diameters (Fig. 9). Nevertheless, as shown by the experimental distribution of diameters, recrystallization plays an important role on the size of the particles. The RS-RDX diameters distribution is narrow compared to that of the other two materials and centers around a diameter of about $600 \mu \mathrm{m}$.

The histogram of the mean, surface-equivalent and volume-equivalent diameters are represented making use of the classes provided by the COULTER device. Our analysis of the segmented volumes does not take into account grains lying on the border of the $\mu \mathrm{CT}$ images which are incompletely segmented. Without correction, the sampling method would be biased toward small particles, as large particles have a higher probability to lie on the border. To correct this bias, use is made of the Miles-Lantuéjoul correction (Lantuéjoul, 1980; Miles, 1974), which assigns to each particle $X$ the weight $1 / P\{X \subset D\}$ where:

$$
P\{X \subset D\}=\frac{\left(L-L_{x}\right)\left(L-L_{y}\right)\left(L-L_{z}\right)}{L^{3}}
$$

is the probability that a particle $X$ is completely included within the volume $D$ (Serra, 1982), $L$ is the size of the volume, and $L_{x}, L_{y}, L_{z}$ are the lengths of the bounding box around particle $X$. Both the original and corrected surface-equivalent diameter histogram are shown in Figs. (9b-d) for information.

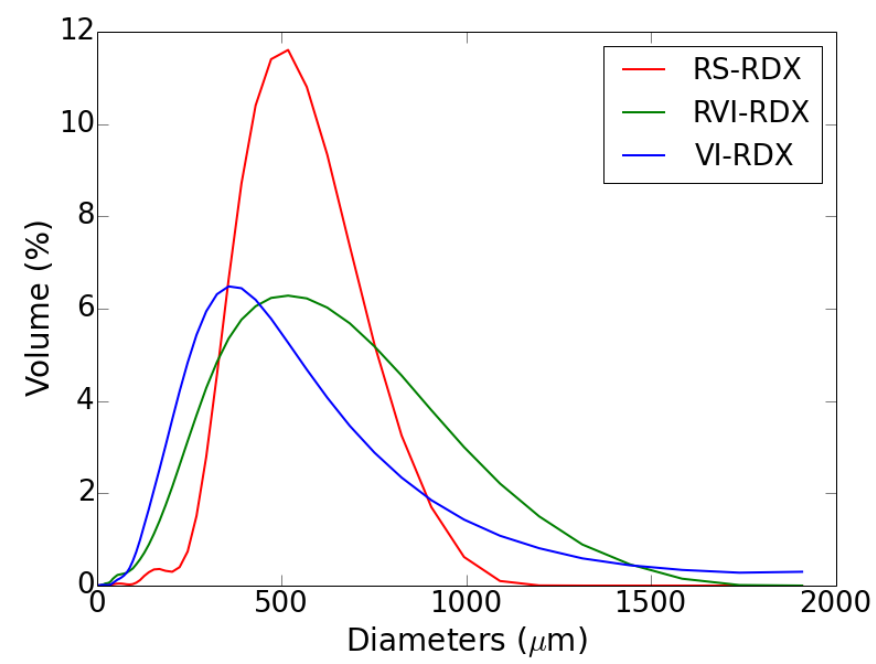

Figure 8: Probability distribution of experimental diameters as measured by the COULTER LS230 SLS device, for the three RDX materials. 


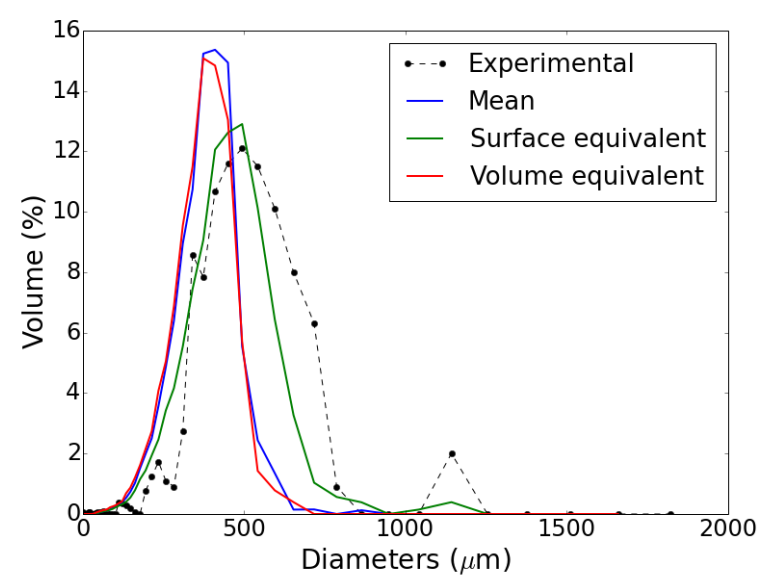

(a)

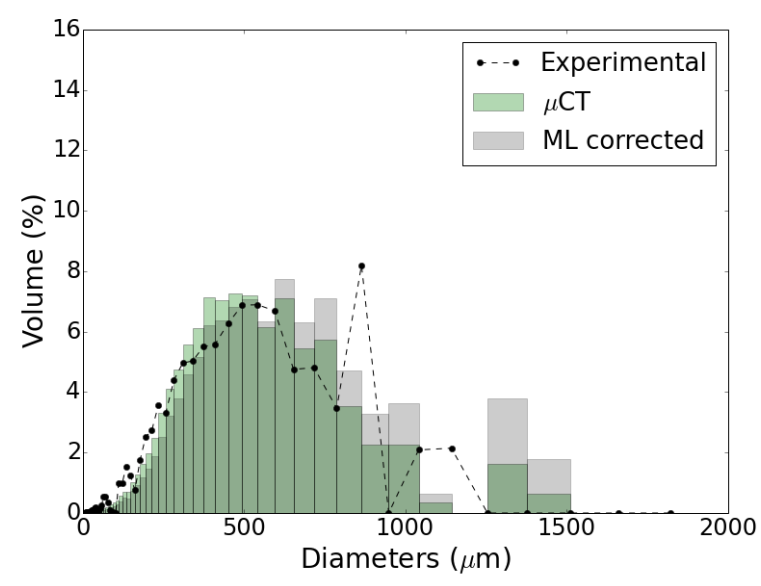

(c)

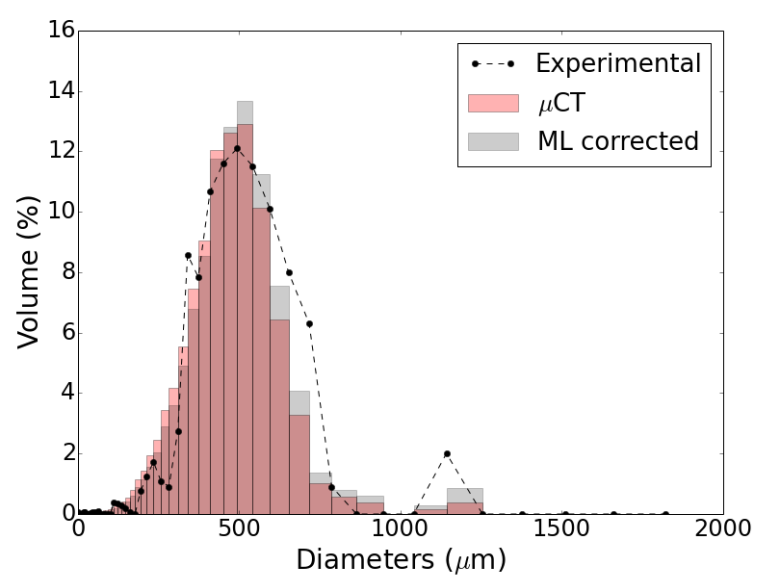

(b)

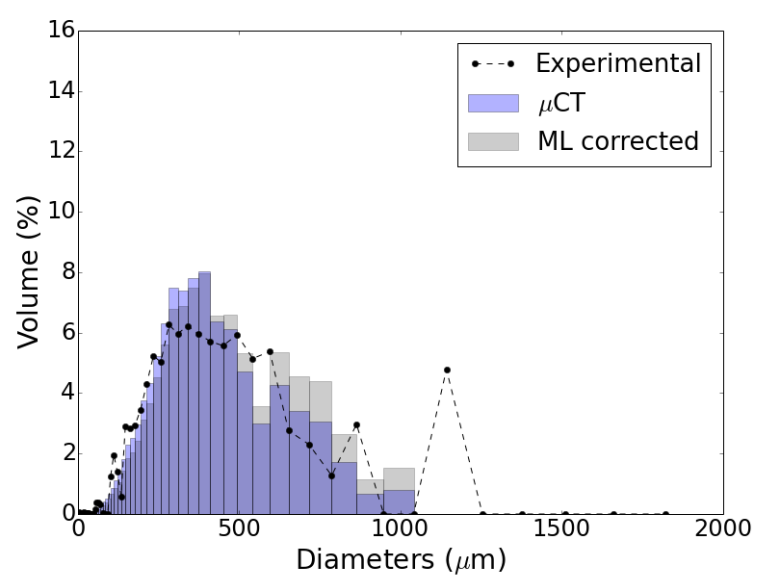

(d)

Figure 9: Histogram of grains diameters: comparison between experimental data and granulometry distribution inferred from image analysis. (a) RS-RDX material (various definition of the diameter). (b-d): Surface-equivalent diameter (RS-RDX, RVI-RDX and VI-RDX resp.).

The surface-equivalent diameter of the RS-RDX particles are bigger than their volume-equivalent and mean diameters, as shown in Fig. (9a) where, for clarity, only the heights of the histogram bins are represented. Similar trends (not shown) are observed for the two other materials. The surface-equivalent diameters, considered in Figs. (9b-d), is closest to the experimental data. Experimental data on figure 9 are raw data without any smoothing exhibiting some spikes. The distribution curve of RS-RDX particles (Fig. 9b), obtained with image analysis, is shifted to the left compared to experimental data, and contains a higher number of small particles than the latter. Recall that RS-RDX particles are often twinned and over-segmented during the segmentation process, therefore not all large particles may be detected in the $\mu \mathrm{CT}$ image. The surface-equivalent distribution of the RVI-RDX and VI-RDX measured by the experimental device and by image analysis are in good agreement with each other (Fig. 9c-d). Differences occur mainly along the 
distribution tail, i.e. for large diameters. These small differences are more in particle sizes that in amount of particles. The histogram is defined in volume, so that the number of particles with a large volume is small and may not be representative. For example, only three particle with a surface-diameter larger than $1100 \mu \mathrm{m}$ are observed in the RVI-RDX library of grains.

\section{Generation of virtual microstructures and elastic response}

In the following, the segmented micro-computed tomography images are used to construct a library of grains to generate virtual microstructures. The virtual microstructures shall have the same volume fraction, morphological granulometry and spatial covariance as measured in the image, but also, the same effective elastic properties. The overall behavior as well as the full-field local elastic response is predicted using the so-called "Fast Fourier Transform" (or "FFT") method, originally proposed by Moulinec and Suquet (1994) for homogenization problems in mechanics. This method, which makes use of 3D microstructure images as input, does not require meshing and is especially useful for treating complex heterogeneous structures. Stress equilibrium and strain admissibility are enforced in the Fourier domain whereas the local constitutive law are computed in the real space. The decomposition of fields into divergence-free and rotational-free parts is provided by the Green operator associated to linear-elastic problems (Mura, 1982), and fast Fourier transforms are used to switch between the two spaces. Over times, refined FFT algorithms and methods have been proposed. The present work employs the "discrete" Green operator introduced by Willot (2015), which improves on the accuracy of the local fields near interfaces. This FFT scheme has notably been applied to homogenization problems related to energetic materials (see e.g. Ambos et al. (2015)). Although FFT and finite element methods have been found to achieve, in general, similar accuracy, it has been argued that the former performs better in terms of memory costs (Gasnier et al., 2018).

\subsection{Generation of representative virtual microstructures}

The Random Sequential Adsorption (RSA) method (Feder, 1980) is used to build virtual structures using the library of grains. In the RSA algorithm, each grain is implanted sequentially, at a random position, until the selected grain is adsorbed. A grain is adsorbed when it does not intersect any previously implanted grains. The library of grains is composed of grains extracted from the micro-computed tomography segmented volumes. Each grain of the library is characterized by several descriptors including volume, surface, size of the minimal

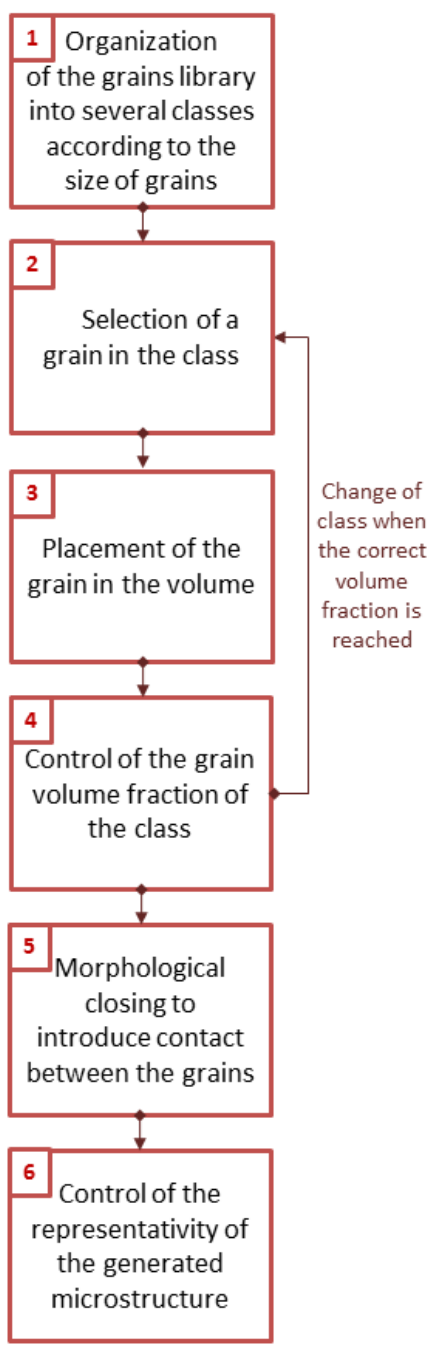

Figure 10: Diagram of the methodology used to generate virtual microstructures 


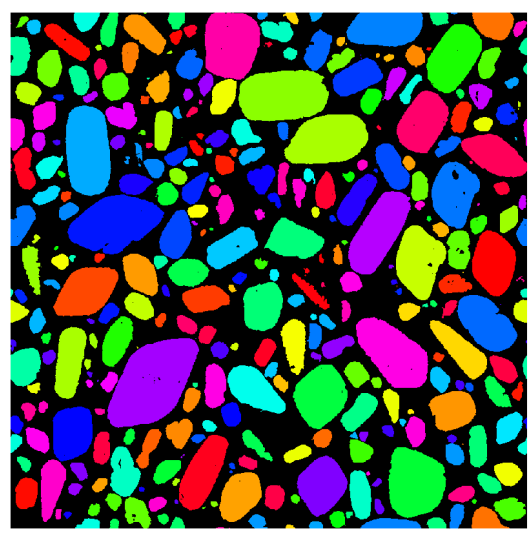

(a)

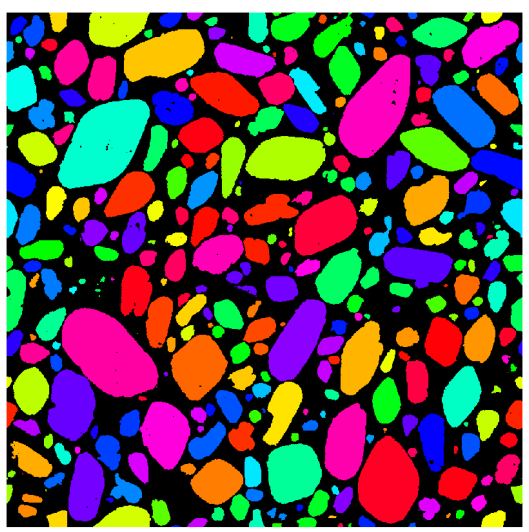

(b)

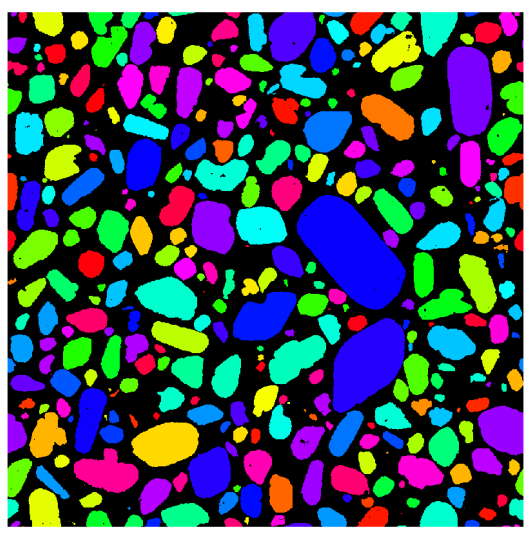

(c)

Figure 11: Slices extracted from $1000^{3}$ voxels volumes of VI-RDX/Wax. (a) Segmented micro-computed tomography. (b-c) Generated microstructures with representative grains (b) and with selected grains (c), which are closer to spheres compared to (a) and (b). These grains have been selected thanks to the sphericity index computed in the library of particles.

oriented bounded box, sphericity indexes and angularity index. Grains not entirely included in the segmented volumes are discarded and are not present in the library of shapes.

In the rest of this study, the library of grains is divided into several classes, according to the volume of the grains (step 1 Fig. 10). We implant the grains sequentially starting with the class which contains the bigger grains, until the class containing the smallest grains is picked. This method allows one to generate packing with moderate grain density (in the present case a volume fraction of $56 \%$ ). The grains can be selected randomly in the class. It is also possible to select grains with specific properties (for exemple a high index of angularity, sphericity, convexity...) as each grain in the library is characterized with several descriptors (step 2 Fig. 10). The next step (step 3 Fig.10) is the implantation of the grain in the volume. For the first 20 grains, random positions are chosen until the grain is adsorbed. For the remaining grains, in case of intersections, the grain is moved in a random direction. If the grain is not adsorbed once all possible positions along the line have been tested, a new random initial position is picked. After 50 trials with the same grain, a new random grain is selected in the class. The volume of grains implanted for each class is controled to respect the granulometry of the real material. These steps are repeated until the correct volume fraction from each class is reached and until the final volume fraction is reached (step 4 Fig. 10). The microstructures generated are periodic. Two examples of generated microtructures containing VI-RDX grains are presented in Fig. (11) and compared with the $\mu \mathrm{CT}$ image. When grains are selected randomly without bias, the resulting microstructures are found to be representative of the real materials in terms of spatial covariance and granulometry (see Figs. 12). This verification is the final step to generate representative virtual microstructures (step 6 Fig. 10).

\subsection{Elastic response of micro-computed tomography volumes}

The elastic response of the material is considered hereafter, with the constitutive law $\sigma(\mathbf{x})=$ $\mathbb{C}(\mathbf{x}): \varepsilon(\mathbf{x})$ in which $\sigma(\mathbf{x})$ is the local stress tensor, $\varepsilon(\mathbf{x})$ the local strain and $\mathbb{C}$ is the stiffness tensor. 


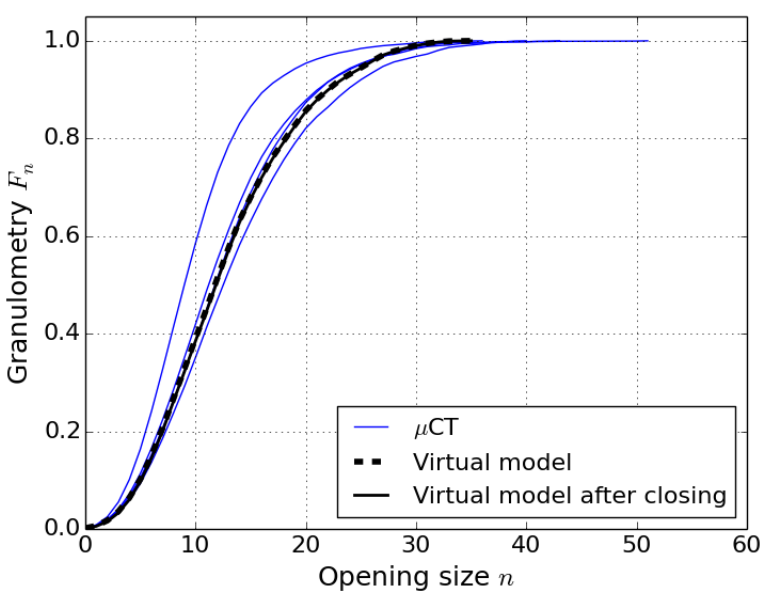

(a)

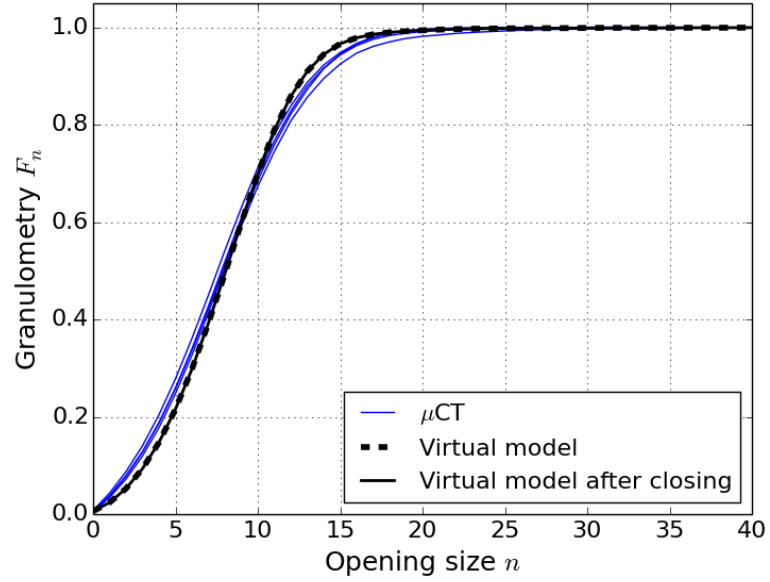

(b)

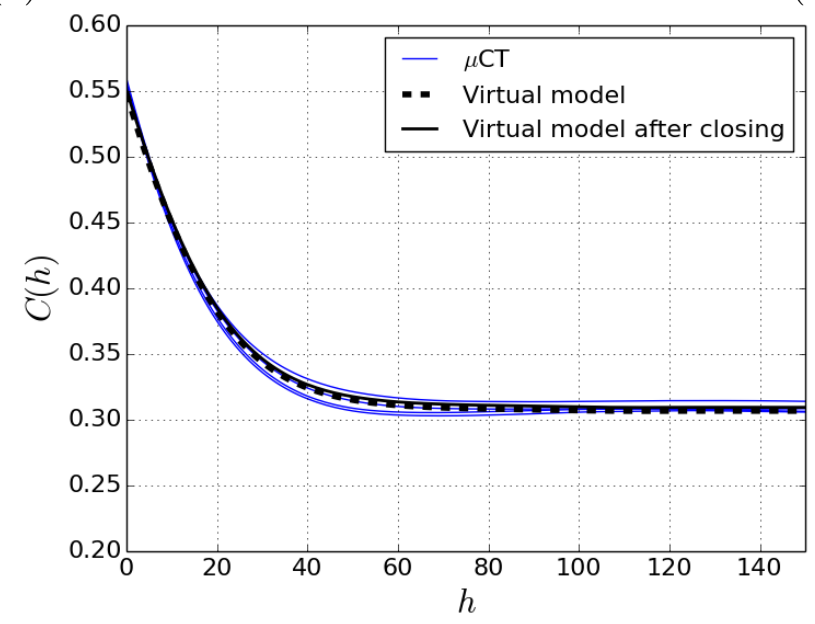

(c)

Figure 12: Comparison between the $\mu \mathrm{CT}$ images and generated model of the VI-RDX material in terms of the morphological granulometry of the grains (a) or binder (b) and their spatial covariance (c). The four $\mu \mathrm{CT}$ images are represented by blue solid lines, the generated microstructure without morphological closing by a black dashed line, and the same microstructure with morphological closing by a black solid line.

Small deformations, quasi-static equilibrium, and periodic boundary conditions are assumed. Wax is assumed to yield an isotropic response with Young modulus 55.7 MPa, Poisson ratio 0.3 (Hossain et al., 2009). RDX grains are orthotropic crystals with moderate anisotropy, so that the stiffness tensor comprises 9 independent moduli. Hereafter we use the experimental moduli determined with Brillouin spectroscopy (Bolme and Ramos, 2014). The crystallographic orientation of the RDX grains are assumed to be random variables uniformly-distributed on the sphere. Hydrostatic compression is applied, i.e. $\langle\operatorname{tr}(\varepsilon(\mathbf{x}))\rangle=1 \%$ is enforced where $\langle\cdot\rangle$ denotes a spatial mean over the unit cell.

The effective bulk modulus $K$, determined using all four segmented volumes of each material, vary greatly according to the type of RDX grains (Tab. 4). The VI-RDX/Wax presents the softest response and is also the less shock-sensitive material (Borne and Beaucamp, 2002). A FFT map 
Table 4: Effective bulk modulus $K$ for the RS-RDX/Wax, RVI-RDX/Wax and VI-RDX/Wax materials.

\begin{tabular}{c|ccc} 
Material & RS-RDX/Wax & RVI-RDX/Wax & VI-RDX/Wax \\
\hline $\mathrm{K}(\mathrm{GPa})$ & 0.471 & 0.561 & 0.389
\end{tabular}

of the stress field in the binder is represented in Fig. (13) for the VI-RDX material. Similar maps are observed for all types of materials. Stress concentration zones appear to be located around contact points or in-between grains which are close to one another, a feature noticed in similar grain packings (Willot et al., 2013). These computations are made on the whole 3D microstructures. Results may not be clearly visible on the 2D slice shown on figure 13. Most grains, including the largest ones, are in contact with a few neighboring grains. The mean connectivity, which corresponds to the mean number of contact point of each grain, is around 2.6 for the three materials. If we only consider grains with a diameter equivalent in volume higher than $585 \mu \mathrm{m}$, the mean connectivity per grain is around 18.4. Likewise, the number of regions around grains where the mean stress is highest, and the intensities of these local maxima, depend on the grain size. This point, which is outside the scope of the present work, is not discussed here.

Such features may be described by means of stress concentration factors that depend on how close to one another the two grains are, and how stiff they are compared to their embedding medium (Hyeonbae et al., 2015) (see also McPhedran et al. (1988) for an earlier work in the context of conductivity). The histogram of the mean stress $(1 / 3) \operatorname{tr}(\sigma(\mathbf{x}))$ values are represented in Fig. (14) for the various $\mu \mathrm{CT}$ samples. The mean stress in the grain is higher in the VI-RDX based material compared to the RS-RDX and RVI-RDX based materials. The former also presents

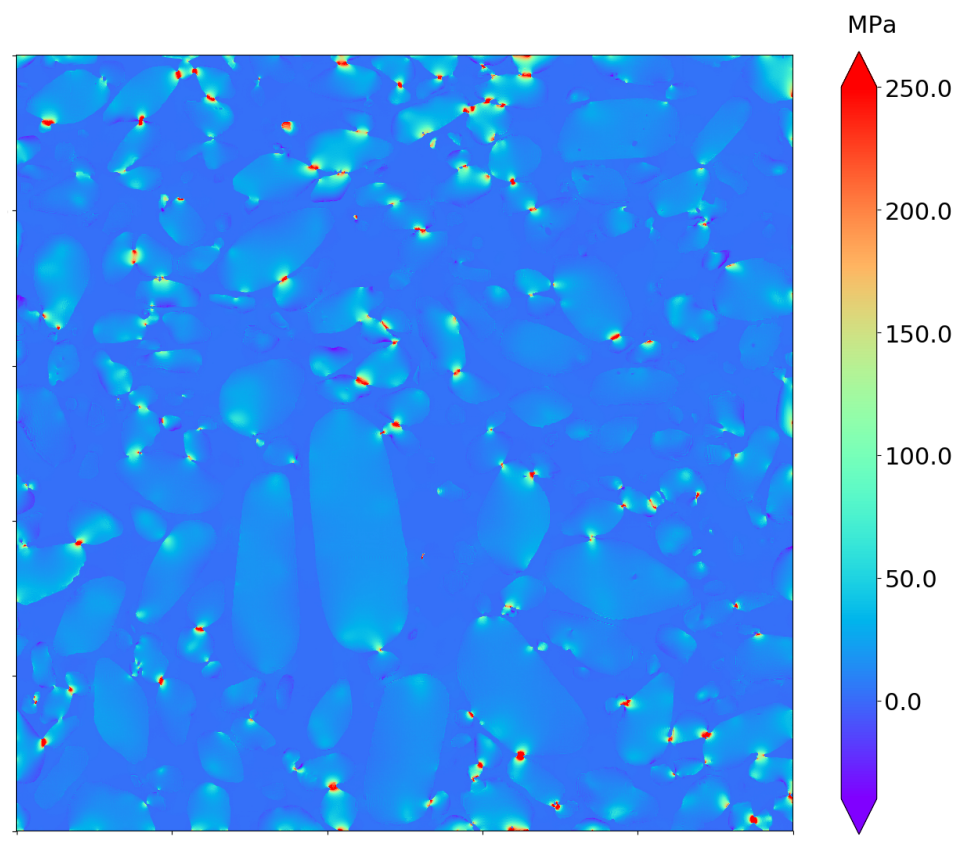

Figure 13: FFT map of the mean stress (opposite of pressure) in MPa along a 2D slice extracted from $\mu \mathrm{CT}$ image of a VI-RDX material. Highest stress values are located along contact points between grains. 
the most narrow stress histogram. The histograms of the stress field in both the grains and wax present asymmetric distribution tails close to powerlaws, features which are consistent with results obtained in elasticity for polycrystals (Willot et al., 2020).

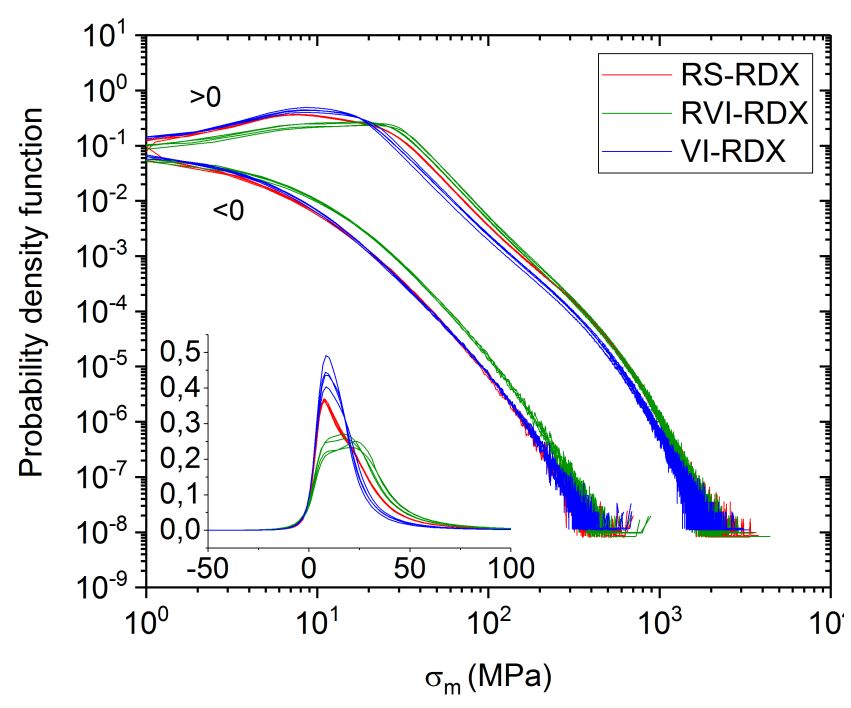

(a)

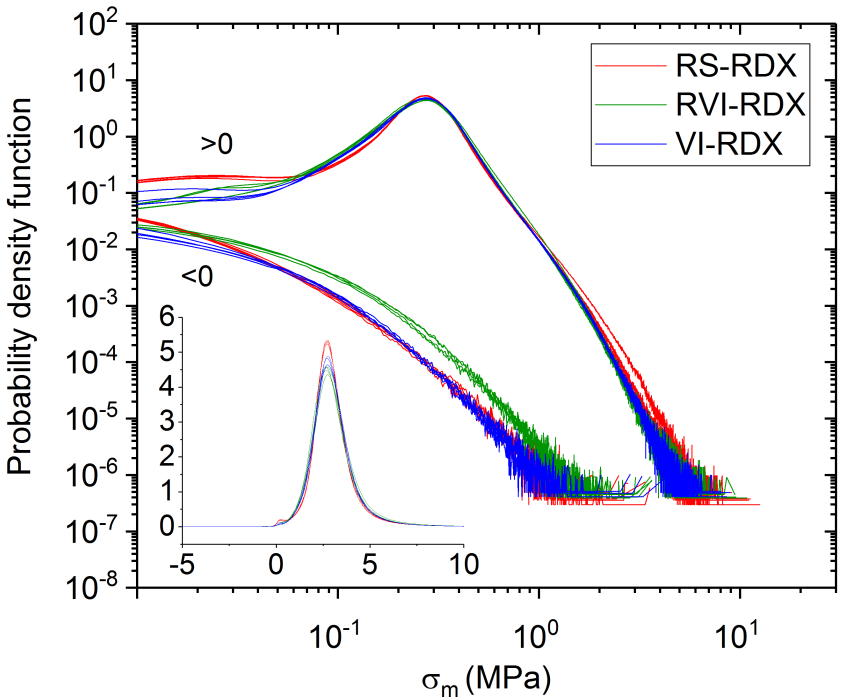

(b)

Figure 14: Log-Log plot of the histogram of the mean stress $(1 / 3) \operatorname{tr}(\sigma(\mathbf{x}))$ in the RDX grains (a) and in the wax binder (b) for the three types of materials. Histograms are computed on several samples of each material: negative parts of the histograms are represented by the curves shown at the bottom of the graphs. Inset (bottom-left): distribution in linear scale.

\subsection{Elastic response of virtual materials}

The same hydrostatic loading is now applied to the virtual microstructures. In this section, we focus on the VI-RDX material. The effective bulk modulus, predicted by Fourier computations, is two times softer than that computed using $\mu \mathrm{CT}$ volumes (Table 5 ). Recall however that the random sequential algorithm forbids grain interpenetration, or even contact points and thus the grains of virtual microstructure have weak connectivity. This feature, which is not apparent in covariance or granulometry curves, is especially important for predicting the mechanical properties since wax is much softer than the RDX grains. Therefore, we seek for a simple way to increase connectivity while leaving nearly intact other properties. A morphological closing (erosion of dilation) with cubic structuring element is employed. This operation fills in narrow gaps in-between grains and increases the contact surfaces between grains. It also, as a side effect, removes the intra-granular defects. Consequently, the intra-granular defects are re-implanted after the closing operation. Changes in the volume fraction, spatial covariance and granulometries are negligible (Figs. 12). The bulk modulus of the microstructure with higher grain connectivity is greatly increased and matches that obtained on the $\mu \mathrm{CT}$ image (Table 5) when a cubic structuring element of size 1 is chosen.

To validate our hypothesis, the histogram of the local hydrostatic stress field is computed for the two virtual microstructures with and without closing, and compared to the stress histogram 
Table 5: Bulk modulus $K$ of the generated microstructures

\begin{tabular}{l|c} 
Type of microstructure & $K$ \\
\hline Mean on the $4 \mu \mathrm{CT}$ volumes & $0.389 \mathrm{GPa}$ \\
Generated microstructure & $0.202 \mathrm{GPa}$ \\
Generated microstructure after a closing & $0.395 \mathrm{GPa}$
\end{tabular}

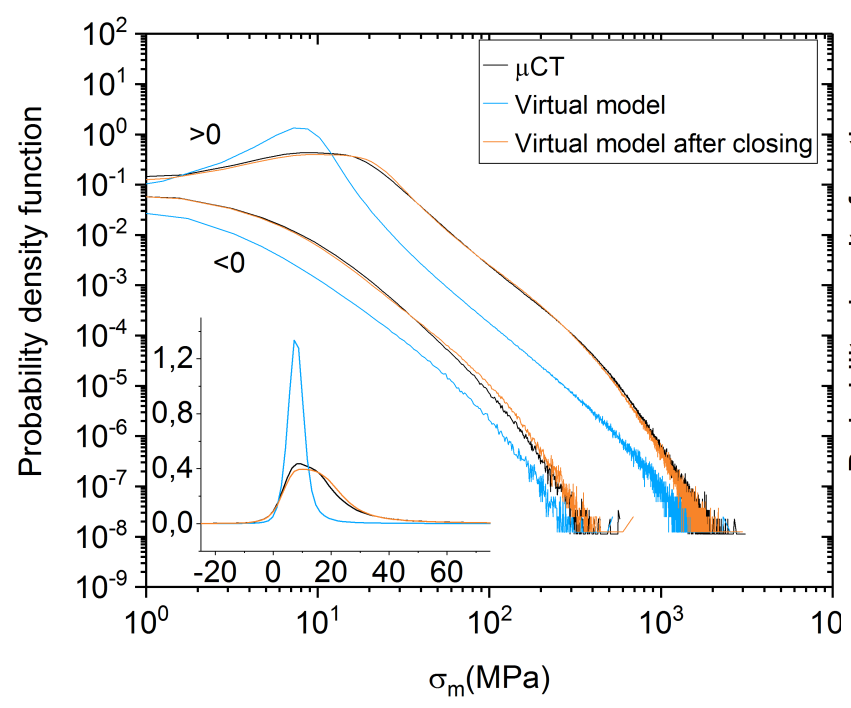

(a)

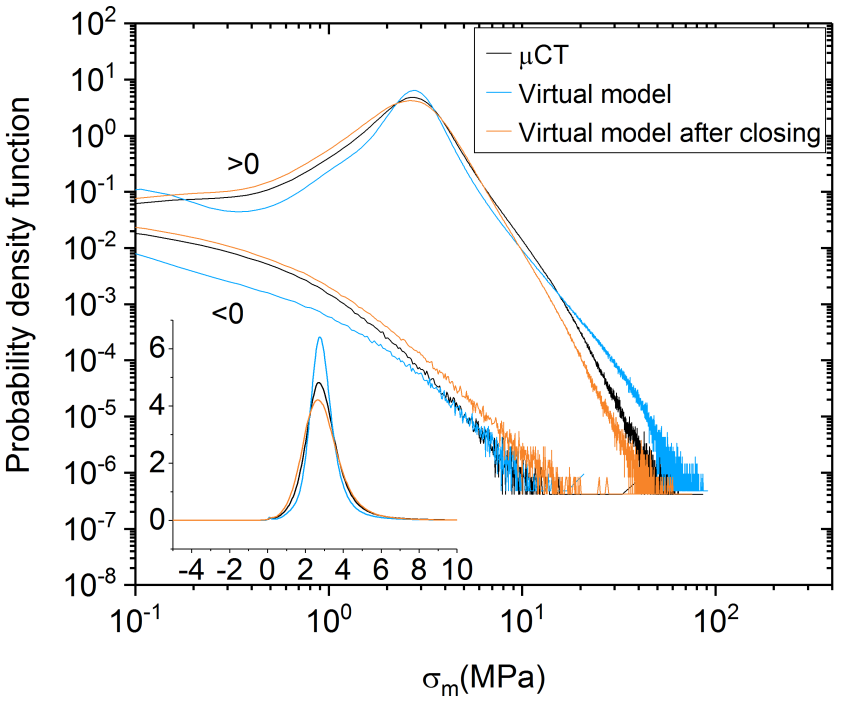

(b)

Figure 15: Mean stresses distributions in the (a) RDX grains and (b) the wax binder for the VI-RDX/Wax microcomputed tomography and generated microstructures. The negative values are also plotted (lowest curves). The distribution on a linear scale is presented on the bottom left.

in the $\mu \mathrm{CT}$ image (Fig. 15). The histogram of the stress field within the virtual microtucture with closing matches that measured on the $\mu \mathrm{CT}$ image, with a very good accuracy. The same stress field histogram is very different in the virtual microstructure when no closing operation has been performed, highlighting the strong effect of grain connectivity with respect to local and effective properties.

Although the grains' connectivity has a strong effect on the effective elastic properties, this is not so for the shape of the grains. Materials made of grains with low or high sphericity were generated using the same methodology (Kaeshammer et al., 2019b). More precisely, we generated materials containing grains with sphericity index lower or higher than the mean value (Kaeshammer et al., 2019a). Their effective elastic bulk modulus, predicted by Fourier computations, was found to be very close to that of the unbiased simulated microstructures. Moreover, this result was unchanged whether a morphological closing was applied or not, or when the angularity was used instead of sphericity. Finally, the stress histograms are found to be quite similar in all cases (see Fig. 16). 


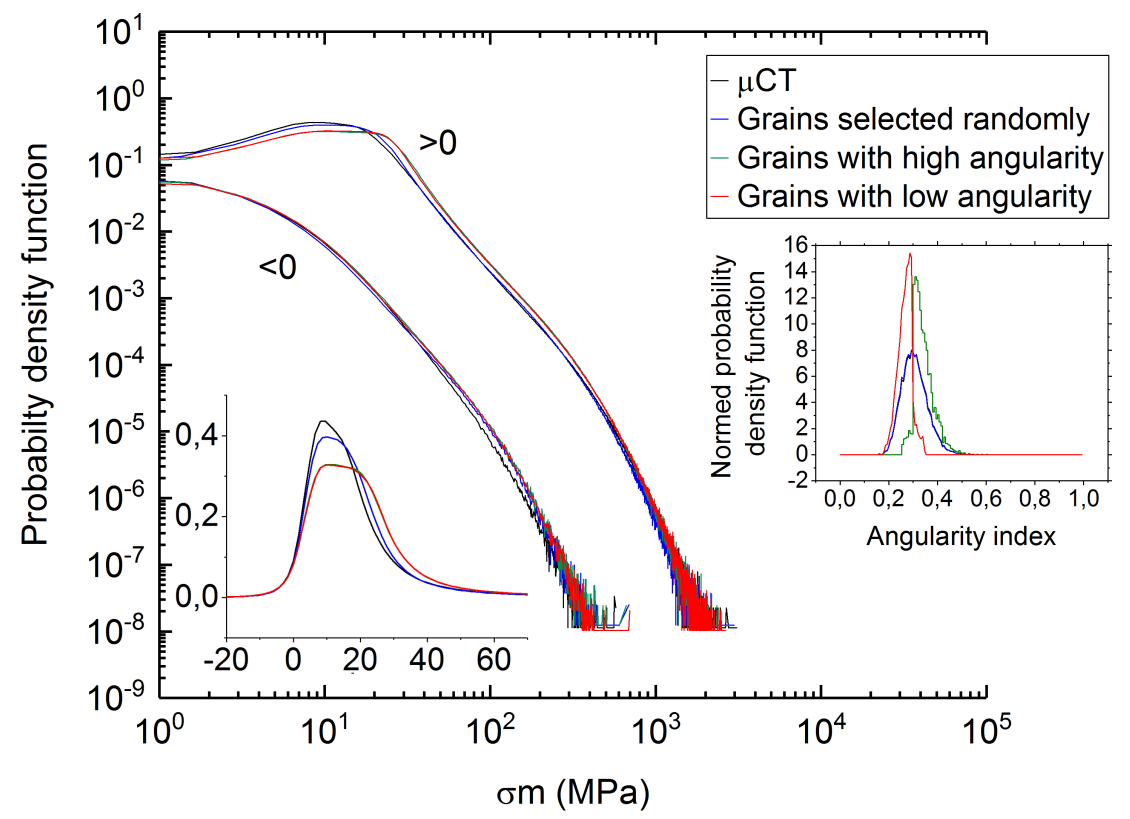

Figure 16: Mean stresses histogram within generated material with grains representative of the VI-RDX grains (black) or grains selected with a bias: low (red) and high angularity (green). The negative values are also plotted (lowest curves). The distribution on a linear scale is presented on the bottom left. The distribution of the angularity index in each microstructure is presented on the figure on the right.

\section{Conclusion}

Three types of materials containing granular structures have been studied. They are composed of RDX grains embedded in a wax matrix. The properties of the constituents are identical for each of the three materials but the shape of the RDX grains, and so the microstructures differ. Micro-computed tomography images of these materials have been segmented. The grain shape and size-distribution, and their spatial distribution have been characterized using morphological means. This analysis reveals that the three materials have an identical grain spatial distribution but a different grain size distribution in addition to different grain shapes. The segmented microcomputed tomography images have been found to be representative of the granular material. This has been demonstrated by an examination of the variance associated to estimates of the grain density, but also by comparison with experimental data, light-scattering measurement and flotation methods, which are similar and validate image analysis results.

We have also shown that the use of micro-computed tomography and image analysis combined with light-scattering measurement and flotation allows computing accurate size and bulk density distribution. Moreover, image analysis techniques combined with the flotation method improves the estimation of the intra-granular defects volume. This is due to the fact that the flotation method allows the detection of small cavities entrapped in the grains with a good accuracy, but is not adapted to detect large cavities in only a few grains. On the contrary, the resolution of the 
micro-computed tomography scanner is not high enough to detect intra-granular defects smaller than $1 \mu \mathrm{m}$, but the analysis of the micro-computed tomography images allows to measure the volume of the biggest intra-granular defects, even in only one grain.

The elastic response of the three materials segmented volumes have been computed and shows noticeable differences. It reveals the importance of the granular structure on the elastic response of a material. Virtual microstructures that mimic the granular structures have been generated using a random sequential adsorption method. These virtual microstructures are representative of the micro-computed tomography images in terms of covariances and other morphological characteristics; however they yield markedly different (softer) elastic properties. We have shown that these differences are induced by the lack of connectivity in the grain packing of the virtual microstructures. A simple method using morphological closing is proposed to increase the grains connectivity. The elastic properties of the granular materials are recovered once the connectivity of the particles packing has been adjusted. Furthermore, the local response in the virtual materials, represented by the stress histograms, are in good agreement with that obtained for the micro-computed tomography images, once the grains connectivity has been corrected. This result highlights how quasi-static mechanical properties of granular structures depend on certain regions of interest in the grain packing, and, conversely, that reconstruction methods, and segmentation, may greatly affect the predictions of mechanical properties.

Credit authorship contribution statement. É. Kaeshammer: investigation, formal analysis, writing - original draft. L. Borne, F. Willot, P. Dokládal \& S. belon: writing - review \& editing.

Acknowledgements. This work was partly supported by the French General Directorate for Armament (DGA) of the Ministry of the Armed Forces. The authors are grateful to P. Rey (CEA Gramat) for its technical contribution to this project. The authors also wish to thank B. Erzar and J. Corbel for useful discussions.

\section{Appendix A. Variance properties of Boolean media with wide granulometry}

Let us consider a Boolean random set (Matheron, 1975) $\mathcal{M}$ made of the union of primary grains $A(\ell)$ of diameter $0<\ell<\infty$. The grains are compact convex (possibly random) sets with centers distributed spatially according to a Poisson point process $\mathcal{P}$ of intensity $\theta(\ell) \mathrm{d} \ell$, representing the mean number of object per unit volume in the range $[\ell ; \ell+\mathrm{d} \ell]$, and accordingly:

$$
\mathcal{M}=\bigcup_{i=0}^{+\infty} \mathcal{M}_{[i \mathrm{~d} \ell,(i+1) \mathrm{d} \ell]}, \quad \mathcal{M}_{[\ell, \ell+\mathrm{d} \ell]}=\bigcup_{x \in \mathcal{P}(\theta(\ell) \mathrm{d} \ell)}[x+A(\ell)],
$$

where $x+A(\ell)$ is the set $A(\ell)$ translated by a vector $x$ and $\mathcal{M}_{[\ell, \ell+\mathrm{d} \ell]}$ is a random set with monodisperse grain and infinitesimal grain density. Thus, set $\mathcal{M}$ is defined by the intensity distribution function $\theta(\ell)$, equivalent to a granulometry distribution, and by the primary grains $A(\ell)$, equivalent to a shape function. We recall the expression for the covariance of the complementary set of the union of two independent random sets $\mathcal{M}_{1}$ and $\mathcal{M}_{2}$ as:

$$
\begin{aligned}
C_{\left(\mathcal{M}_{1} \cup \mathcal{M}_{2}\right)^{c}}(h) & =P\left\{x, x+h \in\left(\mathcal{M}_{1} \cup \mathcal{M}_{2}\right)^{c}\right\} \\
& =P\left\{x, x+h \in \mathcal{M}_{1}^{c}\right\} P\left\{x, x+h \in \mathcal{M}_{2}^{c}\right\}=C_{\mathcal{M}_{1}^{c}}(h) C_{\mathcal{M}_{2}^{c}}(h) .
\end{aligned}
$$


Thus, by extension, the covariance of the complementray set of $\mathcal{M}$ reads:

$$
\begin{aligned}
C_{\mathcal{M}^{c}}(h) & =P\left\{x, x+h \in \mathcal{M}^{c}\right\}=\exp \int_{\ell>0} \log C_{\mathcal{M}_{[\ell, \ell+\mathrm{d} \ell]}}(h) \\
& =\exp \int_{\ell>0}-\mathrm{d} \ell \theta(\ell)[2|A(\ell)|-K(\ell ; h)],
\end{aligned}
$$

where $K(\ell ; h)=|A(\ell) \cap[h+A(\ell)]|$ is the mean geometrical covariogram of $A(\ell)$, averaged over all directions $h$, and $|A(\ell)|$ denotes the volume of the grain $A(\ell)$. Here, use has been made of the Choquet-Matheron-Kendall theorem (see e.g. Chiu et al. (2013)). Let us now assume that the shape functions $A(\ell)$ are homothetic to one another:

$$
A(\ell)=\frac{\ell}{\ell_{0}} A\left(\ell_{0}\right), \quad K(\ell ; h)=\left(\ell / \ell_{0}\right)^{d} K_{0}\left(h \ell_{0} / \ell\right), \quad K_{0}(h)=K\left(\ell_{0} ; h\right)
$$

for some size $\ell_{0}$, where $d$ is the dimension. The mean volume fraction of $\mathcal{M}$ reads:

$$
f=1-\exp \int_{\ell>0}-\mathrm{d} \ell \theta(\ell)|A(\ell)|
$$

Assume that $\mathcal{M}$ contains a proportion of very large grains so that the tail of the granulometry function may be modeled by the power-law:

$$
\theta(\ell) \sim \theta_{0}\left(\ell / \ell_{0}\right)^{\nu}, \quad \ell \rightarrow \infty
$$

with $\theta_{0}$ constant and $\nu<-(d+1)$ so that $f<1$. The use of (A.3) and (A.6) together with (A.5) entails:

$$
C_{\mathcal{M}^{c}}(h)=(1-f)^{2} \exp \int_{\ell>h} \mathrm{~d} \ell \theta(\ell)\left(\frac{\ell}{\ell_{0}}\right)^{d} K_{0}\left(h \frac{\ell_{0}}{\ell}\right),
$$

where the domain of integration accounts for the fact that function $K_{0}(h)$ is zero whenever $h \geq \ell_{0}$. Finally:

$$
\begin{aligned}
C_{\mathcal{M}^{c}}(h)= & (1-f)^{2} \exp \left\{\theta_{0} h^{1+\nu+d} / \ell_{0}^{\nu} I\left(\ell_{0}\right)\right\}, \\
& I\left(\ell_{0}\right)=\frac{1}{\ell_{0}^{d}} \int_{0}^{1} \mathrm{~d} s K_{0}\left(s \ell_{0}\right) s^{-\nu-d-2}<+\infty,
\end{aligned}
$$

as $h \gg 1$. The powerlaw decay of the covariance function $\left(C_{\mathcal{M}^{c}}(h)=C_{\mathcal{M}^{c}}(\infty)+O\left(h^{1+\nu+d}\right)\right)$ may be compared with results obtained in one dimension for time series problems by Koutsoyiannis (2002) who introduced multi-time processes to interpret the "Hurst phenomenon", a slow decay of the autocovariance function for the time series.

We now specialize these results to $d=3$. The variance of the apparent volume fraction, measured on spherical domains of diameter $L$ and volume $V=\pi / 6 L^{3}$ may be computed as (Lantuéjoul, 1991):

$$
D^{2}(V)=\frac{1}{V f(1-f)} \int_{h \leq L} \mathrm{~d} h 4 \pi h^{2}\left[C_{\mathcal{M}(h)}-f^{2}\right],
$$


where $C_{\mathcal{M}}(h)=2 f-1+C_{\mathcal{M}^{c}}(h)$ is the covariance of $\mathcal{M}$. We obtain:

$$
D^{2}(V)=\frac{1-f}{f} \frac{24 \theta_{0} I\left(\ell_{0}\right)}{(\nu+6) \ell_{0}^{\nu}}\left(\frac{\pi / 6}{V}\right)^{-(4+\nu) / 3}+O(1 / V), \quad V \rightarrow \infty
$$

where the first term on the r.h.s. is the leading-order term when $-7<\nu<-4$. With the choice

$\nu=-6$, the volume fraction of grains of size $\gtrsim \ell$ decreases proportionally to $1 / \ell^{2}$ (Eq. A.5), and the variance scales as $D^{2}(V) \sim V^{-2 / 3}$.

\section{References}

Ambos, A., Willot, F., Jeulin, D., Trumel, H., 2015. Numerical modeling of the thermal expansion of an energetic material. International Journal of Solids and Structures 60-61, 125-139.

Baillou, L., Dartyge, J., Spyckerelle, C., Mala, J., 1993. Influence of crystal defects on sensitivity of explosives, in: Tenth Symposium (International) on Detonation, pp. 816-823.

Beucher, S., 1994. Watershed, hierarchical segmentation and waterfall algorithm, in: Viergever, M.A., Serra, J., Soille, P. (Eds.), Mathematical Morphology and its applications to image processing. Dordrecht. volume 2, pp. 69-76.

Beucher, S., Meyer, F., 1993. The morphological approach to segmentation: the watershed transformation. Mathematical morphology in image processing 34, 433-481.

Bolme, C.A., Ramos, K.J., 2014. The elastic tensor of single crystal RDX determined by Brillouin spectroscopy. Journal of Applied Physics 116.

Borne, L., 1993. Influence of intragranular cavities of RDX particles batches on the sensitivity of cast wax bonded explosives, in: Tenth Symposium (International) on Detonation, pp. 286-293.

Borne, L., 1998. Explosive crystal microstructure and schock-sensitivity of cast formulations, in: Eleventh Symposium (International) on Detonation.

Borne, L., 2006. Particles of an explosive of low sensitivity to shock and associated treatment process. US Patent 8,747,581 B2.

Borne, L., Beaucamp, A., 2002. Effects of explosive crystal internal defects on projectile impact initiation, in: Twelth Symposium on Detonation.

Borne, L., Beaucamp, A., 2005. Quantitative characterisation of particle internal defects in particle lots. Measurement Science and Technology 16, 925-930.

Borne, L., Patedoye, J.C., Spyckerelle, C., 1999. Quantitative characterisation of internal defects in rdx crystals. Proppellants, Explosives, Pyrotechnics 24, 255-259.

Borne, L., Patedoye, J.L., 2009. Device for measuring the density of particles by flotation. US Patent 7,607,341 B2. 
Chabardès, T., Dokládal, P., Faessel, M., Bilodeau, M., 2017. An affinity score for grains merging and touching grains separation, in: $13^{\text {th }}$ International Symposium on Mathematical Morphology and Its Applications to Signal and Image Processing, pp. 423-434.

Chabards, T., 2018. Segmentation automatique de matriaux granulaires. Ph.D. thesis. Mines Paristech. Fontainebleau.

Chiu, S.N., Stoyan, D., Kendall, W.S., Mecke, J., 2013. Stochastic geometry and its applications. John Wiley \& Sons, Chichester.

Feder, J., 1980. Random sequential adsorption. Journal of Theoretical Biology 87, 237-254.

Gaiselmann, G., Neumann, M., Schmidt, V., Pecho, O., Hocker, T., Holzer, L., 2014. Quantitative relationships between microstructure and effective transport properties based on virtual materials testing. AIChE Journal 60, 1983-1999.

Gasnier, J., Willot, F., Trumel, H., Jeulin, D., Besson, J., 2018. Thermoelastic properties of microcracked polycrystals. Part I: Adequacy of Fourier-based methods for cracked elastic bodies. International Journal of Solids and Structures 155, 248-256.

Guyon, E., Roux, S., Hansen, A., Bideau, D., Troadec, J.P., Crapo, H., 1990. Non-local and non-linear problems in the mechanics of disordered systems: application to granular media and rigidity problems. Reports on Progress in Physics 53, 373.

Hossain, E., Ketata, C., Islam, R., 2009. Experimental study of physical and mechanical properties of natural and synthetic waxes using uniaxial compressive strenght test.

Hyeonbae, K., Hyundae, L., KiHyun, Y., 2015. Optimal estimates and asymptotics for the stress concentration between closely located stiff inclusions. Mathematische Annalen 363, 1281-1306.

Jeulin, D., 2002. Spatial statistics and micromechanics of materials, in: Morphology of condensed matter, pp. 3-36.

Jeulin, D., 2016. Power laws variance scaling of Boolean random varieties. Methodology and Computing in Applied Probability 18, 1065-1079.

Kaeshammer, E., Dokládal, P., Willot, F., Belon, S., Borne, L., 2019a. Generation of virtual microstructures of energetic materials based on micro-computed tomography images analysis, Fraunhofer ICT, Karlsruhe. 50 ${ }^{\text {th }}$ International Annual Conference of ICT, Energetic Materials: Past, Present and Future, June 25-28. Online at https://hal.archives-ouvertes.fr/ hal-02139566.

Kaeshammer, E., Dokládal, P., Willot, F., Erzar, B., Belon, S., Borne, L., 2019b. A morphological study of energetic materials: analysis of micro-computed tomography images to generate representative microstructures, Af3P, Tours. Europyro 2019. 44 ${ }^{\text {th }}$ International Pyrotechnics Seminar, Tours, France, June 3-7. Online at https://hal .archives-ouvertes.fr/hal-02139567. 
Kaneko, K., Terada, K., Kyoya, T., Kishino, Y., 2003. Global-local analysis of granular media in quasi-static equilibrium. International Journal of Solids and Structures 40, 4043-4069.

Koutsoyiannis, D., 2002. The hurst phenomenon and fractional gaussian noise made easy. Hydrological Sciences Journal 47, 573-595.

Lantuéjoul, C., 1980. On the estimation of mean values in individual of particles. Microscopica Acta 5, 73 .

Lantuéjoul, C., 1991. Ergodicity and integral range. Journal of Microscopy 161, 387-403.

Matheron, G., 1975. Random sets and integral geometry. Wiley, New-York.

Matheron, G., 1989. Estimating and choosing: an essay on probability in practice. Springer Science \& Business Media.

McPhedran, R., Poladian, L., Milton, G., 1988. Asymptotic studies of closely spaced, highly conducting cylinders. Proceedings of the Royal Society of London. A. Mathematical and Physical Sciences 415, 185-196.

Miles, R.E., 1974. On the elimination of edges effects in planar sampling, in: Harding, E.F., Kendall, D.G. (Eds.), Stochastic geometry: a tribute to the memory of Rollo Davidson. Wiley, London, pp. 228-247.

Milton, G.W., 2002. The Theory of Composites. Cambridge Univ. Press, Cambridge.

Mishra, I.B., Vande Kieft, L.J., 1988. Novel Approach to Insensitive Explosives, in: 19th Intenational Annual Conference of ICT, pp. 1-25.

Moulard, H., Delclos, A., Kury, J.W., 1985. The Effects of RDX Particle Size on the Shock Sensitivity of Cast PBX Formulations, in: Eight Symposium (International) on Detonation.

Moulinec, H., Suquet, P., 1994. A fast numerical method for computing the linear and non linear mechanical properties of the composites. Comptes Rendus de l'Académie des Sciences Série II 318, 1417-1423.

Mura, T., 1982. Micromechanics of defects in solids. Martinus Nijhoff Publishers, The Hague.

Neumann, M., Stenzel, O., Willot, F., Holzer, L., Schmidt, V., 2020. Quantifying the influence of microstructure on effective conductivity and permeability: virtual materials testing. International Journal of Solids and Structures 184, 211-220.

Peña, A., McNamara, S., Lind, P., Herrmann, H., 2009. Avalanches in anisotropic sheared granular media. Granular Matter 11, 243-252.

Russ, J., Dehoff, R., 2012. Practical stereology. Springer Science \& Business Media.

Serra, J., 1982. Image Analysis and Mathematical Morphology. Academic Press, Inc., Orlando, FL, USA. 
Stenzel, O., Pecho, O., Holzer, L., Neumann, M., Schmidt, V., 2016. Predicting effective conductivities based on geometric microstructure characteristics. AIChE Journal 62, 1834-1843.

Stoyan, D., Kendall, W.S., Mecke, J., 1995. Stochastic geometry and its applications. John Wiley \& Sons, Chichester. Second edition.

Torquato, S., 2013. Random heterogeneous materials: microstructure and macroscopic properties. volume 16. Springer Science \& Business Media, New-York, USA.

van der Steen, A.C., Verbeek, H.J., Meulenbrugge, J.J., 1989. Influence of RDX crystal shape on the shock sensitivityof PBXs, in: Ninth Symposium on Detonation.

Wang, H., Pietrasanta, A., Jeulin, D., Willot, F., Faessel, M., L., S., Moreaud, M., 2015. Modeling of mesoporous alumina microstructure by $3 \mathrm{D}$ random models of platelets. Journal of Microscopy 260, 287-301.

Willot, F., 2015. Fourier-based schemes for computing the mechanical response of composites with accurate local fields. Comptes Rendus Mécanique 343, 232-245.

Willot, F., 2017. Mean covariogram of cylinders and applications to Boolean random sets. Journal of Contemporary Mathematical Analysis 52, 305-315.

Willot, F., Brenner, R., Trumel, H., 2020. Elastostatic field distributions in polycrystals and cracked media. Philosophical Magazine 100, 661-687.

Willot, F., Gillibert, L., Jeulin, D., 2013. Microstructure-induced hotspots in the thermal and elastic responses of granular media. International Journal of Solids and Structures 50, 16991709 .

Wu, S., Yu, B., Wu, Z., Fang, S., Shi, B., Yang, J., 2018. Effect of particle size distribution on the electrochemical performance of micro-sized silicon-based negative materials. RSC advances $8,8544-8551$.

Xu, W., Sun, H., Chen, W., Chen, H., 2018. Transport properties of concrete-like granular materials interacted by their microstructures and particle components. International Journal of Modern Physics B 32, 1840011.

Yang, Q., 2012. Recursive Bilateral Filtering, in: Computer Vision - ECCV 2012, Springer, Berlin, Heidelberg. pp. 399-413. 